The Astrophysical Journal, 524:295-310, 1999 October 10

(c) 1999. The American Astronomical Society. All rights reserved. Printed in U.S.A.

\title{
TIME EVOLUTION OF THE MAGNETIC ACTIVITY CYCLE PERIOD. II. RESULTS FOR AN EXPANDED STELLAR SAMPLE
}

\author{
STEVEN H. SAAR \\ Harvard-Smithsonian Center for Astrophysics, MS-58, 60 Garden Street, Cambridge, MA 02138; saar@cfa.harvard.edu
}

AND

AXEL BRANDENBurg

Department of Mathematics, University of Newcastle upon Tyne, NE1 7RU, United Kingdom; Axel.Brandenburg@newcastle.ac.uk Received 1998 November 18; accepted 1999 May 11

\begin{abstract}
We further explore nondimensional relationships between the magnetic dynamo cycle period $P_{\text {cyc }}$, the rotational period $P_{\text {rot }}$, the activity level (as observed in Ca II HK), and other stellar properties by expanding the stellar sample studied in the first paper in this series. We do this by adding photometric and other cycles seen in active stars and the secondaries of CV systems and by selectively adding less certain cycles from the Mount Wilson HK survey; evolved stars, long-term HK trends and secondary $P_{\text {cyc }}$ are also considered. We confirm that most stars with age $t \gtrsim 0.1 \mathrm{Gyr}$ occupy two roughly parallel branches, separated by a factor of $\sim 6$ in $P_{\text {cyc }}$, with the ratio of cycle and rotational frequencies $\omega_{\text {cyc }} / \Omega \propto \mathrm{Ro}^{-0.5}$, where Ro is the Rossby number. Using the model of the first paper in this series, this result implies that the $\alpha$ effect increases with mean magnetic field (contrary to the traditional $\alpha$ quenching concept) and that $\alpha$ and $\omega_{\text {cyc }}$ decrease with $t$. Stars are not strictly segregated onto one or the other branch by activity level, though the high- $\omega_{\mathrm{cyc}} / \Omega$ branch is primarily composed of inactive stars. The expanded data set suggests that for $t \gtrsim 1 \mathrm{Gyr}$, stars can have cycles on one or both branches, though among older stars, those with higher (lower) mass tend to have their primary $P_{\text {cyc }}$ on the lower (upper) $\omega_{\text {cyc }} / \Omega$ branch. The Sun's $\sim 80$ yr Gleissberg cycle agrees with this scenario, suggesting that longterm activity "trends" in many stars may be segments of long $\left(P_{\text {cyc }} \sim 50-100\right.$ yr) cycles not yet resolved by the data. Most very active stars ( $P_{\text {rot }}<3$ days) appear to occupy a new, third branch with $\omega_{\text {cyc }} / \Omega \propto$ $\mathrm{Ro}^{0.4}$. Many RS CVn variables lie in a transition region between the two most active branches. We compare our results with various models, discuss their implications for dynamo theory and evolution, and use them to predict $P_{\text {cyc }}$ for three groups: stars with long-term HK trends, stars in young open clusters, and stars that may be in Maunder-like magnetic minima.
\end{abstract}

Subject headings: novae, cataclysmic variables - stars: activity - stars: late-type stars: magnetic fields - stars: rotation

\section{INTRODUCTION}

Ever since the first detection of cyclic magnetic behavior in solar-like stars (e.g., Wilson 1978), there has been great interest in using the results to understand better the dynamo mechanism believed responsible. Several groups have searched the available cycle data derived from Mount Wilson Ca II $S$ measurements for correlations between cycle periods $\left(P_{\text {cyc }}\right)$ and amplitudes, and stellar properties such as rotation and convection zone depth (e.g., Noyes, Weiss, \& Vaughan 1984b; Baliunas \& Vaughan 1985; Tuominen, Rüdiger, \& Brandenburg 1988; Saar \& Baliunas 1992; Soon, Baliunas, \& Zhang 1993). After some initially discouraging results (Baliunas \& Vaughan 1985), the recent availability of updated cycle data based on $25 \mathrm{yr}$ of observations (Baliunas et al. 1995, hereafter Bea95) has led to renewed interest in $P_{\text {cyc }}$ relationships (Baliunas et al. 1996a; Ossendrijver 1997).

Recently, Brandenburg, Saar, \& Turpin (1998, hereafter Paper I) studied a nondimensional parameterization of dynamo and activity quantities. They explored relationships between the ratio of cycle and rotational frequencies $\omega_{\text {cyc }} / \Omega\left(=P_{\text {rot }} / P_{\text {cyc }}\right)$, the inverse Rossby number $\mathrm{Ro}^{-1}$ $\left(\equiv 2 \tau_{c} \Omega=4 \pi \tau_{c} / P_{\text {rot }}\right.$, where $\tau_{c}$ is the convective turnover or correlation time; see, e.g., Noyes et al. 1984a, hereafter Nea84), and the fractional $\mathrm{Ca}$ II HK emission flux above background, $R_{\mathrm{HK}}^{\prime}\left(\equiv F_{\mathrm{HK}}^{\prime} / \sigma T_{\text {eff }}^{4} ;\right.$ Nea84). They found a dis- tinct segregation of young and old stars into two approximately parallel bands, with old stars showing $\omega_{\text {cyc }} / \Omega$ values $\approx 6$ times larger than young stars, but both groups showing similar power-law relationships with $\mathrm{Ro}^{-1}$ and $R_{\mathrm{HK}}^{\prime}$. The results implied $\omega_{\text {cyc }} / \Omega$ evolves in time $t$, decreasing approximately as $t^{-0.35}$, but showing a sharp increase around $t \sim 2-3$ Gyr when the stars seemed to jump from the young to the old branch. The nondimensional parameterization also led to fits with reduced scatter. Interpretation of the correlations using simple dynamo models, together with certain plausible assumptions, revealed that their positive slopes imply an $\alpha$ effect increasing with mean magnetic field $B$, contrary to the typical assumption of $\alpha$-quenching (e.g., $\alpha \propto B^{-2}$ ).

Several questions remained unanswered in Paper I. Many stars in the Mount Wilson sample show evidence for two cycle periods, long-term trends, or cycles plus trends (Bea95). Others show low, nonvariable $S$ values and may be in a state analogous to the solar Maunder minimum (Baliunas \& Jastrow 1990). Still others show high variable activity levels with no clear periodicities. How do these stars fit into the above scenario? What forms do dynamos take in rapid rotators (where the $\mathrm{Ca}$ II diagnostic is ineffective because of chromospheric saturation)? We explore these issues by gathering $(\S 2)$ and analyzing $(\S 3)$ an expanded database of cycle, rotation, and activity data. We discuss the 
results in $\S 4$, where we present new scenarios for cycle evolution, compare with previous studies, and use the results to predict $P_{\text {cyc }}$ for selected stars where cycles have not yet been observed. A summary is given in $\S 5$.

\section{THE EXPANDED STELLAR SAMPLE}

Paper I studied the cycles of a sample of 22 stars, the $P_{\text {cyc }}$ values of which had "false-alarm probabilities" (FAP values) of FAP $\leq 10^{-5}$, equivalent to FAP "grades" of good or excellent. Our strategy in this paper is to add carefully additional stars with less certain cycles and/or trends from the Mount Wilson database and other sources. Our hope is that the increased numbers and enlarged parameter range spanned by the additional stars could overcome possible increases in scatter caused by the reduced certainty of their $P_{\text {cyc }}$ values, permitting us to define the cycle relationships better and to answer some of the questions noted above. Since we selected the new stars primarily based on the visual appearance of their time series and how consistent these seemed with the derived $P_{\text {cyc }}$ or trends, however, there is clearly a risk of introducing selection bias. But while each added star is thus a "judgment call" and open to criticism, we will see that the basic results are robust and not significantly altered over a variety of different star classifications, selections, and weighting schemes (§ 3.1).

Unfortunately, errors in the main variables we are studying are dominated by poorly understood or quantified systematic errors. Measurements of $P_{\text {rot }}$ are affected by differential rotation (e.g., Donahue, Saar, \& Baliunas 1996) and those of $P_{\text {cyc }}$ by active region growth and decay (Donahue, Dobson, \& Baliunas 1997), fundamental varia-

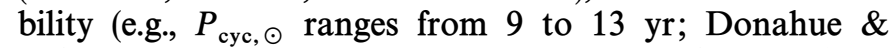
Baliunas 1992), and errors due to the finite length of the data record. Both are likely affected by the assumption of a purely sinusoidal variation in the period analysis (Bea95). Because it is difficult to assign accurate errors for these effects, we cannot properly weight the data. Weights were instead assigned qualitatively, as a gauge of the reliability of a given $P_{\text {cyc }}$. The best detections (good or excellent FAP grades, very clear photometric cycles) were given a weight $w=4$, fair grades/moderately clear $P_{\text {cyc }} w=2$, and so on. A variety of factors could reduce this initial grade (by a factor of 2 per case), such as very weak or intermittent cycle amplitude, variable $P_{\text {cyc }}$, disagreement on $P_{\text {cyc }}$ among different studies, or use of a cycle "clock" less firmly tied to magnetic activity (e.g., radial velocity residuals in binaries). Stars significantly evolved from the main sequence were also given lower $w$ owing to less accurate $\tau_{c}$ estimates (see $\S 2.4$ ). These factors are discussed in more detail below for the specific cases.

\subsection{Selected Additional Mount Wilson Survey Stars}

Starting with the sample of $P_{\text {cyc }}$ with good or excellent FAP grades from Paper I, we first considered adding Wilson survey stars from Bea95 with FAP grades of at least "fair" (numerically, FAP $\leq 10^{-2}$ ), plus stars with apparent long-term trends $(=\mathscr{L})$. Secondary cycle periods $\left(P_{\mathrm{cyc}}^{(2)}\right)$ with FAP grades fair or better were also considered, if the primary period was graded at least fair. To these we added a few stars that appeared to have trends or cycles but were not so classified in Bea95 (these were given lower weight). We also used plots in Radick et al. (1998, hereafter Rea98), which extend the time series of several stars to $\sim 30 \mathrm{yr}$, to confirm, and in a few cases, modify the Bea95 results. We consider "irregular variables" $(\mathscr{V})$ and "flat activity" $(\mathscr{F})$ stars in $\S 4$.

From this initial group, we excluded stars with nearly equal masses in unresolved binaries (HD 114378 and HD 3443) since the stars' individual contributions to $S$ are muddled. We also rejected most stars with $S$ time series of less than $12 \mathrm{yr}$ because of unclear or possibly incomplete cycles (a few exceptions are discussed below). The cycles, trends, and other stellar data are given in Table 1 . We discuss the stars individually below, detailing reasons for each star's inclusion or exclusion from the final database, and any changes or additions to $P_{\mathrm{cyc}}$ or the variability classification. We note that many of the excluded $P_{\text {cyc }}$ nonethless agree with the trends found in $\S 3$.

Our visual inspection of the HK time series agreed with the "fair" FAP cycles and/or long-term trends found by Bea95 in HD 1835, 3651, 17925, 18256, 20630, 25998, 26913, 32147 , 35296, 39587, 76151, 101501, 124850, 131156B, 141004, 154417, 176095, 187013, and 224930 (HD 3651, labeled $b$, and $32147=g$, were already included in Paper I; here we add their long-term trends). We note, though, that in HD 18256 (labeled C) and HD 76151 (I), $P_{\text {cyc }}$ seemed somewhat variable, the fractional cycle amplitude in HD 26913 (F) is quite low, and in HD 154417 (Q), the cycle appears only in half of the data record. HD $176095(\beta)$ could be classified $\mathscr{V}$ instead.

Some cases were less clear. HD 100180 (i) exhibits maxima in 1970,1981, 1985, and 1988.5, while weak minima appear in 1976.5, 1983, and 1987 (we visually identify extrema to within $\pm 0.5 \mathrm{yr}$, typically). Thus, the $\left\langle P_{\text {cyc }}\right\rangle=$ $3.6 \mathrm{yr}$ (FAP grade fair) seems confirmed. The evidence seems weaker, though, for the similarly graded $P_{\mathrm{cyc}}^{(2)}=12.9$ yr, basically confined to similar declines from larger maxima in 1968-1972 and 1981-1983. Since the both the earliest data and the data in 1978-1980 are sparse and have larger errors, the identification of this period seems less certain.

HD $187691(o)$ shows weak local $S$ maxima in 1970.5, 1977.5, and $1987.5( \pm 1)$, and local minima in 1974, 1978.5, 1984.5, and (perhaps) 1990. Separation of successive extrema (several not clearly identified) thus average close to the $P_{\text {cyc }}=5.4 \mathrm{yr}$ in Bea95 (graded fair), but with a large ( \pm 1 yr) dispersion. We regard this $P_{\text {cyc }}$ as less certain.

Inspection of the following stars and/or the literature suggested some modification of the Bea95 results was advisable. The cycle identified for HD 136202 (Donahue 1996; $k$ ), not listed in Bea95, seems solid. However, new $P_{\text {cyc }}$ suggested for HD 22049 ( 5 yr; Gray \& Baliunas 1995) and HD 10700 ( 11 yr; Gray \& Baliunas 1994) seemed dubious in light of the nondetection of the former by Donahue (1996) and heavy reliance on less accurate data from 1980 for HD 10700. Long-term trends were added to HD 115404, 155886, 160346, and 201091, all of which show significant trends in $\langle S\rangle$ (cycle amplitude modulation). HD 219834A, whose cycle was deemed dubious in Paper I, was reclassified as an $\mathscr{L}$ star (the primary $\langle S\rangle$ maximum is unmatched, suggesting a full cycle is not yet complete). Long-term trends were rejected for HD 9562 (in agreement with Donahue 1996, who deems it an $\mathscr{F}$ star), 29645, 33608, 107213, 143761, and 212754. In all these cases, the trend amplitude seems negligible, or so small as to be unreliably detected.

HD 165341A (S) appears to have a large amplitude cycle with $P_{\text {cyc }} \sim 15.5$ yr (with peaks in $\sim 1969.5$ and a probable 
TABLE 1

Stellar Properties for Wilson Survey Stars

\begin{tabular}{|c|c|c|c|c|c|c|c|c|c|c|c|c|c|}
\hline Branch, ${ }^{a}$ Symbol & HD & $B-V$ & Spectral Type & $M_{V}$ & $\log \left\langle R_{\mathrm{HK}}^{\prime}\right\rangle$ & $\begin{array}{c}\tau_{\mathrm{c}, \mathrm{E}} \\
\text { (days) }\end{array}$ & $\begin{array}{c}\tau_{\mathrm{c}, \mathbf{T}} \\
\text { (days) }\end{array}$ & $\begin{array}{c}P_{\text {rot }} \\
\text { (days) }\end{array}$ & $\begin{array}{l}P_{\text {cyc }} \\
(\mathrm{yr})\end{array}$ & $\begin{array}{l}P_{\mathrm{cyc}}^{(2)} \\
(\mathrm{yr})\end{array}$ & $\begin{array}{l}P_{\mathrm{cyc}}^{(\mathscr{L})} \\
(\mathrm{yr})\end{array}$ & $w$ & Notes $^{b}$ \\
\hline \multicolumn{14}{|c|}{ Active Stars $\left(\log \left\langle R_{\mathrm{HK}}^{\prime}\right\rangle>-4.75\right)$ with Better Determined Behavior } \\
\hline A,A.. & 1835 & 0.66 & $\mathrm{G} 2.5 \mathrm{~V}$ & 4.85 & -4.433 & 12.3 & 13.4 & 7.78 & 9.1 & $>25$ & $?$ & 2 & $f$ \\
\hline$?, \mathrm{~B} \ldots$. & 17925 & 0.87 & $\mathrm{~K} 2 \mathrm{~V}$ & 5.97 & -4.311 & 21.0 & 20.0 & 6.76 & $>25$ & $\ldots$ & $?$ & $\ldots$ & \\
\hline $\mathrm{A}, \mathrm{C} \ldots \ldots$ & 18256 & 0.45 & F5 IV-V & 2.83 & -4.722 & 3.5 & 1.5: & 3 & 6.8 & $\ldots$ & $\ldots$ & 1 & $\mathrm{f}, 0$ \\
\hline A,D ................... & 20630 & 0.68 & G5 V & 5.03 & -4.420 & 13.3 & 14.2 & 9.24 & 5.6 & $>25$ & $?$ & 2 & $\mathrm{f}$ \\
\hline ?,E ............... & 25998 & 0.52 & F7 V & 3.88 & -4.401 & 6.0 & 6.4 & 3 & $>25$ & $\ldots$ & $?$ & $\ldots$ & \\
\hline $\mathrm{A}, \mathrm{F} \ldots \ldots \ldots \ldots \ldots$ & 26913 & 0.70 & G3 V & 5.34 & -4.391 & 14.2 & 14.9 & 7.15 & $7.8 ?$ & $>25$ & $?$ & 1 & f \\
\hline ?,G ................ & 35296 & 0.52 & F8 V & 4.17 & -4.378 & 6.4 & 6.9 & 3.56 & $>25$ & $\ldots$ & $\ldots$ & $\ldots$ & \\
\hline$?, \mathrm{H} \ldots .$. & 39587 & 0.59 & G0-V & 4.70 & -4.426 & 9.0 & 10.0 & 5.36 & $\ldots$ & $>25$ & $?$ & $\ldots$ & $\mathscr{V}$ \\
\hline I,I .............. & 76151 & 0.67 & G3 V & 4.85 & -4.659 & 12.8 & 13.8 & 15 & 2.52 & $>25$ & $?$ & 2 & $\mathrm{f}$ \\
\hline $\mathrm{A}, \mathrm{J} \ldots \ldots \ldots \ldots$ & 78366 & 0.60 & G0 V & 4.54 & -4.608 & 9.5 & 10.6 & 9.67 & 12.2 & $5.9 ?$ & $\ldots$ & $2,1^{\mathrm{c}}$ & $\mathrm{g}, \mathrm{f}, 1$ \\
\hline$?, \mathrm{~K} \ldots$ & 101501 & 0.72 & G8 V & 5.41 & -4.546 & 15.1 & 15.6 & 16.68 & $\ldots$ & $>25$ & $?$ & $\ldots$ & $\mathscr{V}$ \\
\hline$A, L \ldots$ & 114710 & 0.58 & F9.5 V & 4.42 & -4.745 & 8.6 & 9.5 & 12.35 & 16.6 & 9.6 & $\ldots$ & $2,2^{\mathrm{c}}$ & $\mathrm{g}, \mathrm{f}, 1$ \\
\hline A,M ............ & 115404 & 0.94 & $\mathrm{~K} 1 \mathrm{~V}$ & 6.24 & -4.480 & 22.9 & 22.1 & 18.47 & 12.4 & $>25$ & $?$ & 2 & $\mathrm{~g}, 1,2$ \\
\hline$?, \mathrm{~N} \ldots \ldots \ldots \ldots \ldots$ & 131156B & 1.17 & $\mathrm{~K} 4 \mathrm{~V}$ & $\ldots$ & -4.424 & 25.0 & 28.9 & 11.94 & $>25$ & $\ldots$ & $?$ & $\ldots$ & \\
\hline $\mathrm{A}, \mathrm{O} \ldots$ & 149661 & 0.84 & $\mathrm{~K} 2 \mathrm{~V}$ & 5.82 & -4.583 & 20.0 & 19.1 & 21.07 & 16.2 & 4 & $\ldots$ & 2,2 & $\mathrm{~g}, \mathrm{~g}, 1$ \\
\hline A,P .. & 152391 & 0.76 & G7 V & 5.50 & -4.448 & 16.9 & 16.8 & 11.43 & 10.9 & $\ldots$ & $\cdots$ & 4 & $\begin{array}{c}\text { e, } 3 \\
\text { ent }\end{array}$ \\
\hline$A, Q \ldots \ldots \ldots \ldots \ldots$ & 154417 & 0.57 & $\mathrm{~F} 8 \mathrm{~V}$ & 4.46 & -4.533 & 8.1 & 9.0 & 7.78 & $7.4 ?$ & $\ldots$ & $\ldots$ & 1 & $\mathrm{f}, 4$ \\
\hline $\mathrm{A}, \mathrm{R} \ldots \ldots \ldots \ldots \ldots$ & 156026 & 1.16 & $\mathrm{~K} 5 \mathrm{~V}$ & 7.45 & -4.662 & 25.0 & 28.5 & 21 & 21.0 & $\ldots$ & $\ldots$ & 4 & $\mathrm{e}, 3$ \\
\hline $\mathrm{A}, \mathrm{S} \ldots \ldots \ldots \ldots \ldots$ & $165341 \mathrm{~A}$ & 0.86 & $\mathrm{~K} 1 \mathrm{~V}$ & 5.50 & -4.548 & 20.6 & 19.7 & 19.9 & 15.5 & 5.1 & $\ldots$ & 2,2 & $\mathrm{n}, \mathrm{f}, 5$ \\
\hline$?, \mathrm{~T} \ldots .$. & 190007 & 1.17 & $\mathrm{~K} 4 \mathrm{~V}$ & 6.87 & -4.692 & 25.0 & 28.9 & 28.95 & $13.7 ?$ & $\ldots$ & $\ldots$ & $1^{\mathrm{c}}$ & $\mathrm{f}$ \\
\hline \multicolumn{14}{|c|}{ Inactive Stars $\left(\log \left\langle R_{\mathrm{HK}}^{\prime}\right\rangle \leq-4.75\right)$ with Better Determined Behavior } \\
\hline $\mathrm{I}, a \ldots \ldots \ldots \ldots \ldots$ & Sun & 0.66 & G2 V & 4.83 & -4.901 & 11.9 & 13.0 & 26.09 & 10.0 & 84 & 44 & 4,4 & e,n,6 \\
\hline $\mathrm{I}, b \ldots \ldots \ldots \ldots \ldots$ & 3651 & 0.85 & $\mathrm{~K} 0 \mathrm{~V}$ & 5.65 & -4.991 & 20.3 & 19.4 & 44 & 14.6 & $>25$ & 83 & 4 & $\mathrm{~g}, 1$ \\
\hline $\mathrm{I}, c \ldots \ldots \ldots \ldots \ldots$ & 4628 & 0.88 & $\mathrm{~K} 2 \mathrm{~V}$ & 6.38 & -4.852 & 21.3 & 20.3 & 38.5 & 8.6 & $\ldots$ & $\ldots$ & 4 & $\mathrm{e}, 3$ \\
\hline $\mathrm{I}, d \ldots \ldots \ldots \ldots \ldots$ & 10476 & 0.84 & K1 V & 5.87 & -4.912 & 20.0 & 19.1 & 35.2 & 9.6 & $\ldots$ & $\ldots$ & 4 & $\mathrm{e}, 3$ \\
\hline $\mathrm{I}, e \ldots \ldots \ldots \ldots \ldots$ & 16160 & 0.98 & K3 V & 6.50 & -4.958 & 23.8 & 23.2 & 48.0 & 13.2 & $\ldots$ & $\ldots$ & 4 & $\mathrm{e}, 3$ \\
\hline $\mathrm{I}, f \ldots \ldots \ldots \ldots \ldots$ & 26965 & 0.82 & $\mathrm{~K} 1 \mathrm{~V}$ & 5.92 & -4.872 & 19.2 & 18.4 & 43 & 10.1 & $\ldots$ & $\ldots$ & 4 & $\mathrm{e}, 3$ \\
\hline $\mathrm{I}, g \ldots \ldots \ldots \ldots \ldots$ & 32147 & 1.06 & K5 V & 6.50 & -4.948 & 24.8 & 25.4 & 48.0 & 11.1 & $>25$ & 82 & 4 & $\mathrm{e}, 1$ \\
\hline $\mathrm{I}, h \ldots \ldots \ldots \ldots \ldots$ & 81809 & $0.80:$ & $\mathrm{K} 0 \mathrm{~V}$ & $\ldots$ & -4.921 & 18.5 & 17.9 & 40.2 & 8.2 & $\ldots$ & $\ldots$ & 2 & $e, 3,7$ \\
\hline $\mathrm{I}, i \ldots \ldots \ldots \ldots \ldots \ldots$ & 100180 & 0.57 & F7 V & 4.46 & -4.922 & 8.1 & 9.0 & 14 & 3.6 & $12.9 ?$ & 22 & 2,1 & $\mathrm{f}, \mathrm{f}$ \\
\hline $\mathrm{I}, j \ldots \ldots \ldots \ldots \ldots \ldots$ & 103095 & 0.75 & G8 V & 6.61 & -4.896 & 16.4 & 16.5 & 31 & 7.3 & $\ldots$ & $\ldots$ & 4 & $\mathrm{e}, 3$ \\
\hline $\mathrm{A}, k \ldots \ldots \ldots \ldots \ldots$ & 136202 & 0.54 & F8 IV-V & 3.08 & -5.088 & 6.8 & 4.9: & $16^{\mathrm{d}}$ & 23 & $\ldots$ & $\cdots$ & 1 & $\mathrm{n}, 0$ \\
\hline$?, l \quad \ldots \ldots \ldots \ldots \ldots$ & 141004 & 0.60 & G0 V & 4.07 & -5.004 & 9.5 & 10.6 & 25.8 & $>25$ & $\ldots$ & 50 & $\ldots$ & \\
\hline $\mathrm{I}, m \ldots \ldots \ldots \ldots \ldots$ & 160346 & 0.96 & $\mathrm{~K} 3-\mathrm{V}$ & 6.38 & -4.795 & 23.4 & 22.7 & 36.4 & 7.0 & $>25$ & 52 & 4 & $\mathrm{e}, 1,2$ \\
\hline $\mathrm{I}, n \ldots \ldots \ldots \ldots \ldots$ & 166620 & 0.87 & $\mathrm{~K} 2 \mathrm{~V}$ & 6.15 & -4.955 & 21.0 & 20.0 & 42.4 & 15.8 & $\ldots$ & $\ldots$ & 4 & $\mathrm{e}, 3$ \\
\hline$?, o \ldots \ldots \ldots \ldots \ldots$ & 187691 & 0.55 & F8 IV-V & 3.68 & -5.026 & 7.1 & 5.8: & 10 & $5.4 ?$ & $\ldots$ & $\ldots$ & $1^{\mathrm{c}}$ & $\mathrm{f}, 0$ \\
\hline $\mathrm{I}, p \ldots \ldots \ldots \ldots \ldots$ & 190406 & 0.61 & G $1 \mathrm{~V}$ & 4.56 & -4.797 & 10.0 & 11.1 & 13.94 & 2.6 & 16.9 & 19 & 2,2 & $f, g$ \\
\hline $\mathrm{I}, q \ldots \ldots \ldots \ldots \ldots$ & 201091 & 1.17 & $\mathrm{~K} 5 \mathrm{~V}$ & 7.49 & -4.764 & 25.0 & 28.9 & 35.37 & 7.3 & $>25$ & 46 & 4 & $\mathrm{e}, 1,2$ \\
\hline $\mathrm{I}, r \ldots \ldots \ldots \ldots \ldots$ & 201092 & 1.37 & K7 V & 8.32 & -4.891 & 25.0 & 41.3 & 37.84 & 10.5 & $\ldots$ & $\ldots$ & 2 & $\mathrm{~g}, 1,8$ \\
\hline $\mathrm{I}, s \quad \ldots \ldots \ldots \ldots \ldots$ & 219834B & 0.91 & $\mathrm{~K} 2 \mathrm{~V}$ & $\ldots$ & -4.944 & 22.1 & 21.2 & 43 & 10.0 & $\ldots$ & $\ldots$ & 4 & $\mathrm{e}, 3$ \\
\hline$?, t \ldots \ldots \ldots \ldots \ldots \ldots$ & 224930 & 0.67 & G3 V & 5.33 & -4.875 & 12.8 & 13.8 & 33 & $>25$ & $\ldots$ & 57 & $\ldots$ & \\
\hline \multicolumn{14}{|c|}{ Active Stars $\left(\log \left\langle R_{\mathrm{HK}}^{\prime}\right\rangle>-4.75\right)$ with Less Certain Behavior } \\
\hline A?,U ............. & 22049 & 0.88 & $\mathrm{~K} 2 \mathrm{~V}$ & 6.18 & -4.455 & 21.3 & 20.3 & 11.68 & $5 ?$ & $>25$ & $?$ & 0 & $\mathrm{n}, \mathscr{V}, 9,10$ \\
\hline$?, \mathrm{~V} \ldots \ldots \ldots \ldots \ldots$ & 30495 & 0.63 & G1 V & 4.87 & -4.511 & 10.9 & 12.1 & 10.8 & $\ldots$ & $>25 ?$ & $?$ & $\ldots$ & $\mathscr{V}, 2$ \\
\hline $\mathrm{A} ?, \mathrm{~W} \ldots \ldots \ldots$ & 75332 & 0.52 & F7 V & 3.93 & -4.464 & 6.0 & 6.4 & 4 & $3.5 ?$ & $\ldots$ & $\ldots$ & 1 & $\mathrm{n}$ \\
\hline A $? \ldots \ldots \ldots \ldots$ & 82885 & 0.77 & G8 V & 5.15 & -4.638 & 17.3 & 17.1 & 18.6 & $7.9 ?$ & $\ldots$ & $\ldots$ & 0 & $\mathrm{f}, \mathscr{V} ?, 0,11$ \\
\hline $\mathrm{A} ? \ldots \ldots \ldots \ldots$ & 88737 & 0.56 & F5 IV & 2.45 & -4.622 & 7.7 & 4.9: & $8^{\mathrm{d}}$ & $21 ?$ & $\ldots$ & $\ldots$ & 0 & $\mathrm{n}, 0,9$ \\
\hline $\mathrm{A}, \mathrm{X} \ldots \ldots \ldots \ldots \ldots$ & 98230B & $0.68:$ & G5 V & $\ldots$ & -4.311 & 13.3 & 14.2 & 3.98 & 2.2: & $\ldots$ & $\ldots$ & 1 & $\mathrm{n}, 12$ \\
\hline $\mathrm{A} ? \ldots \ldots \ldots \ldots \ldots$ & 115383 & 0.58 & G0 V & 3.92 & -4.443 & 9.0 & 10.0 & 3.33 & $4.3 ?$ & $\ldots$ & $\ldots$ & 0 & $\mathrm{n}, \mathscr{V}, 9$ \\
\hline$?, Y \ldots \ldots \ldots \ldots \ldots$ & 120136 & 0.48 & F7 IV-V & 3.53 & -4.731 & 4.5 & 3.9 & 3.3 & $0.32 ?$ & $\ldots$ & $\ldots$ & $?$ & $\mathrm{n}, 0,13$ \\
\hline$?, \mathrm{Z} \ldots \ldots \ldots \ldots \ldots$ & 124850 & 0.54 & F7 IV & 2.42 & -4.671 & 6.8 & 4.0: & $4^{d}$ & $>25$ & $\cdots$ & $\cdots$ & $\ldots$ & 0,10 \\
\hline$A ? \ldots \ldots \ldots \ldots \ldots$ & 155885 & 0.86 & $\mathrm{~K} 1 \mathrm{~V}$ & 5.44 & -4.559 & 20.6 & 19.7 & 21.11 & 5.7 & $\ldots$ & $\ldots$ & 0 & $\mathrm{p}, 9$ \\
\hline $\mathrm{A} ?, \alpha \ldots \ldots \ldots \ldots$ & 155886 & 0.86 & $\mathrm{~K} 1 \mathrm{~V}$ & 5.44 & -4.570 & 20.6 & 19.7 & 20.69 & $5 ?$ & $>25$ & $?$ & 0 & $\mathrm{n}, \mathscr{V}, 9,10$ \\
\hline $\mathrm{A} ?, \beta \ldots \ldots \ldots \ldots$ & 176095 & 0.46 & F5 IV & 2.45 & -4.671 & 3.8 & $1.2:$ & $4^{\mathrm{d}}$ & $>25 ?$ & $\ldots$ & $\ldots$ & $\ldots$ & $\mathrm{n}, \mathscr{V} 0,10$ \\
\hline$A ? \ldots \ldots \ldots \ldots$ & 194012 & 0.51 & F5 V & 4.08 & -4.720 & 5.6 & 5.8 & 7 & 16.7 & 5.4 & $\ldots$ & 0 & $\mathrm{p}, \mathrm{f}, 9,11$ \\
\hline $\mathbf{A} ?, \gamma \ldots \ldots \ldots \ldots$ & 206860 & 0.59 & G0 V & 4.64 & -4.416 & 9.0 & 9.6 & 4.86 & 6.2 & $>25 ?$ & $?$ & 0 & $\mathrm{p}, \mathrm{n}, 2,9$ \\
\hline \multicolumn{14}{|c|}{ Inactive Stars $\left(\log \left\langle R_{\mathrm{HK}}^{\prime}\right\rangle \leq-4.75\right)$ with Less Certain Behavior } \\
\hline$I ? \ldots \ldots \ldots \ldots \ldots$ & 10700 & 0.72 & G8 V & 5.68 & -4.958 & 15.1 & 15.6 & 34 & $11 ?$ & $\ldots$ & $\ldots$ & 0 & $\mathrm{n}, \mathscr{F}, 9$ \\
\hline$? \ldots \ldots \ldots \ldots \ldots \ldots$ & 126053 & 0.63 & G3 V & 5.02 & -4.957 & 10.9 & 12.1 & $22^{\mathrm{d}}$ & $14 ?$ & $\ldots$ & $\ldots$ & 0 & $\mathrm{n}, 9$ \\
\hline
\end{tabular}


TABLE $1-$ Continued

\begin{tabular}{|c|c|c|c|c|c|c|c|c|c|c|c|c|c|}
\hline Branch, ${ }^{\mathrm{a}}$ Symbol & HD & $B-V$ & Spectral Type & $M_{V}$ & $\log \left\langle R_{\mathrm{HK}}^{\prime}\right\rangle$ & $\begin{array}{c}\tau_{\mathrm{c}, \mathrm{E}} \\
\text { (days) }\end{array}$ & $\begin{array}{c}\tau_{\mathrm{c}, \mathbf{T}} \\
\text { (days) }\end{array}$ & $\begin{array}{c}P_{\text {rot }} \\
\text { (days) }\end{array}$ & $\begin{array}{l}P_{\mathrm{cyc}} \\
(\mathrm{yr})\end{array}$ & $\begin{array}{l}P_{\mathrm{cyc}}^{(2)} \\
(\mathrm{yr})\end{array}$ & $\begin{array}{c}P_{\mathrm{cyc}}^{(\mathscr{L})} \\
(\mathrm{yr})\end{array}$ & $w$ & Notes $^{b}$ \\
\hline $\mathrm{I}, u$. & 161239 & 0.65 & G5 IV & 2.81 & -5.164 & 11.9 & 16: & 29.2 & $5.7 ?$ & $\ldots$ & $\ldots$ & 0.5 & $\mathrm{f}, 0$ \\
\hline $\mathrm{I}, v \ldots \ldots \ldots \ldots \ldots$ & 185144 & 0.79 & $\mathrm{~K} 0 \mathrm{~V}$ & 5.87 & -4.832 & 18.1 & 17.6 & 29 & $6.5:$ & $\ldots$ & $\ldots$ & 1 & $\mathrm{u}, 14$ \\
\hline $\mathbf{A} ?, w \ldots \ldots \ldots \ldots$ & 187013 & 0.47 & F5 V & 3.40 & -4.795 & 4.1 & 3.3 & $8^{\mathrm{d}}$ & $25 ?$ & $>25$ & $\cdots$ & 0 & $\mathrm{n}, 9,10$ \\
\hline$?, x \ldots \ldots \ldots \ldots \ldots$ & 219834A & 0.80 & G5 IV & 3.62 & -5.066 & 18.5 & 38: & 42 & $>25$ & $\ldots$ & $70:$ & $\ldots$ & $0,2,11$ \\
\hline
\end{tabular}

${ }^{a}$ Branch for primary $P_{\text {cyc }}: \mathrm{A}=$ active, I = inactive; symbol used in right-hand panels of Figs. 1, 2, and 5.

b NoTes.- p, f, g, e, u, n: FAP grades (Bea95) "poor," "fair," "good," "excellent," "ungraded," "new." $\mathscr{V}:$ variable. $\mathscr{F}:$ flat activity. 0: modified $\tau_{\mathrm{c}, \mathrm{T}}$ and/or lum. class based on new $\pi, M_{V} .1: P_{\text {cyc }}$ in Paper I, $P_{\text {cyc }}^{(2)}$ or $\mathscr{L}$ (i.e., $P_{\text {cyc }}^{(2)}>25$ yr) from Bea95 added here. $2:$ new $\mathscr{L}$ (i.e., $P_{\text {cyc }}>25$ yr) added. $3:$ in Paper I, no additions/changes. 4: $P_{\text {cyc }}$ in only part of time series. 5: old $P_{\text {cyc }} \rightarrow P_{\mathrm{cyc}}^{(2)}$. 6: Gleissberg $P_{\mathrm{cyc}}^{(2)}$ (Wolff 1976). 7: approx. sp. type, $B-V$ of secondary star (see Paper I). 8: $P_{\text {cyc }}$ modified based on Rea98. 9: tentative $P_{\text {cyc }}$ (new or "poor") rejected. 10: added $\mathscr{L}$ (i.e., $P_{\text {cyc }}>25$ yr) from Bea95. 11: "fair" or better Bea95 $P_{\text {cyc }}$ rejected (see text). 12: photometric $P_{\text {cyc }}$ (Strassmeier et al. 1997), $\langle S\rangle$ from Rutten 1987, $\tau_{c}$ based on estimated $B-V$. 13: anomalously short $P_{\text {cyc }}$, may reflect active region growth/decay, not included in branch fit. 14: HK record short, $P_{\text {cyc }}$ underestimated?

${ }^{c}$ Not typically included in a branch fit.

${ }^{\mathrm{d}} P_{\text {rot }}$ estimated from $\left\langle R_{\mathrm{HK}}^{\prime}\right\rangle$ (Bea96a).

one in 1980), not identified in Bea95 perhaps because the second proposed maximum falls in a time interval with little data. We took this new period as the "primary" and shifted the Bea95 period $\left(P_{\mathrm{cyc}}=5.1 \mathrm{yr}\right)$ to $P_{\mathrm{cyc}}^{(2)}$.

HD $201092(r)$ has a rather irregular $P_{\text {cyc }}$ variation. The new Rea98 data suggest a shorter $P_{\text {cyc }} \approx 10.5$ yr may be a better fit (maxima in $1969 \pm 1,1979.5 \pm 1$, and 1990).

Maxima in $S$ for HD 82885 appear in $1970,1976,1983$ 1986, 1990 (weak), 1992.5, and 1994, minima in 1975, 1978 1980, 1989, and 1993.5. The spacing between these extrema do not match either the $P_{\text {cyc }}=7.9 \mathrm{yr}$ (fair) or the $P_{\mathrm{cyc}}^{(2)}=13$ $\mathrm{yr}$ (poor) periods in Bea95 very well. We reject this star.

The HK trace of HD 157856 shows a local minimum in $\sim 1983-1984$ and a maximum in 1988-1989 with almost no other significant feature. The fair $P_{\mathrm{cyc}}=15.9 \mathrm{yr}$ would imply another minimum around 1968 and maximum in $\sim 1973$; a very weak maximum in $\sim 1970.5$ and minimum in 1971-1972 do not agree with this period, and no other significant feature is apparent. We neglected this star.

HD $161239(u)$ exhibits maxima in 1974, 1978.5, 1984.5, 1989.5, and 1994.5 and minima in 1968, 1976, 1982, 1987.5, and 1992.5, yielding $\left\langle P_{\text {cyc }}\right\rangle \sim 5.7$ yr (Bea95; fair FAP) but with significant scatter. The expected maximum at $\sim 1968$ 1969 is also missing; we give this star lower weight.

The "poor" $P_{\text {cyc }}=16.7 \mathrm{yr}$ of HD 194012 looks more convincing (maxima in 1967.5 and 1984.5) than the fair $P_{\mathrm{cyc}}^{(2)}=5.4 \mathrm{yr}$. The uncertainty led us to ignore this star.

Two stars with tentative $P_{\text {cyc }}$ without FAP grades were also considered. The $P_{\text {cyc }}=24$ ? yr given for HD 88737 looks too long; maxima in $1968.5( \pm 1.5)$ and 1989.5 suggest $P_{\text {cyc }} \sim 21 \mathrm{yr}$, but this too seems uncertain. We could find no evidence for the ungraded $P_{\mathrm{cyc}}=22$ ? yr cycle in HD 126053; weak maxima in 1969 and 1982.5 and minima in 1974-1977 and 1988-1990 are more consistent with $P_{\mathrm{cyc}} \approx$ $14 \mathrm{yr}$. We dropped this star because of the $P_{\text {cyc }}$ uncertainty. We also considered, but ultimately rejected, cycles for HD $115383\left(P_{\mathrm{cyc}} \sim 4.3\right.$ ? yr, peaks in $1970.5,1975,1979.5,1984$, 1988,1992 plus $\mathscr{L}$ ?), and HD $155886\left(P_{\mathrm{cyc}} \sim 5\right.$ ? yr, peaks in 1972.5, 1977.5, 1982.5, and 1987.5, plus $\mathscr{L}$ ), HD 187013 $\left(P_{\mathrm{cyc}} \sim 25\right.$ ? yr, similar to HD 136202), and HD 206860 and HD 155885 (poor FAPs, possibly caused due to uncorrected, weak, long-term trends).

HD $120136(\mathrm{Y})$ is an unusual case of an extremely short $P_{\text {cyc }}=0.32$ yr (Maulik, Donahue, \& Baliunas 1997).
Although these authors reject it as a "true" cycle period, its position in Figures 1 and 2 lies only $\sim 0.2$ dex above the I branch. We include it in the plots, but not in the fits, owing to the uncertainty over the origin of the period. Another unusual case is HD 98230B ( $\xi$ UMa B $=X$ ), which we include in Table 1 though its $P_{\text {cyc }}$ is based on photometry (Strassmeier et al. 1997) since its cycle and rotational properties appear to place it firmly in the company of other active Mount Wilson stars ( $\$ 3.1$; Figs. 1 and 2).

HD 75332 (W, $\mathscr{V}$ in Bea95) shows fairly regular modulation in a cubic spline through its seasonal averages (Rea98), suggesting $P_{\mathrm{cyc}} \approx 3.5 \mathrm{yr}$ (e.g., maxima: 1970, 1973, 1976.5, $1980,1983,1987$, and 1991.5). We tentatively add it with a low weight. The Rea98 data also appear to confirm a cycle in HD $185144(v)$.

Finally, we have added the $\sim 80$ yr Gleissberg (1971) cycle as a $P_{\text {cyc }}^{(2)}$ for the Sun $(a)$. We adopt $P_{\text {cyc, } \odot}^{(2)}=84 \mathrm{yr}$ (Wolff 1976), though values ranging from 76 to $\sim 100 \mathrm{yr}$ can be found in the literature. The existence of a long $P_{\mathrm{cyc}}^{(2)}$ in the Sun has important implications for the interpretation of long-term "trends" (see $\S \S 4$ and 4.3).

We now turn to $P_{\text {cyc }}$ determined from long-term photometry of more active stars; as such, the periods refer to cycles in starspots rather than in plage/network.

\subsection{Active Spotted Dwarfs}

In active, rapidly rotating stars, emission from the chromosphere reaches a maximum "saturated" level (e.g., Vilhu 1984), and thus Ca II HK ceases to be an effective diagnostic of magnetic variability. Variability caused by starspots, however, continues to increase beyond this rotation rate (O'Dell et al. 1995; Krishnamurthi et al. 1998) perhaps because the ratio of spot to plage area continues to increase (Radick, Lockwood, \& Baliunas 1990; Foukal 1998). Thus photometric variability may still be reliable tracers of cycles even at high activity levels. We discuss 14 spotted dwarf systems with photometric $P_{\mathrm{cyc}}$ estimates below; references for $P_{\text {cyc }}$ can be found in Table 2 .

Cycle periods were primarily determined from photometry, although some (see Table 2) came from cyclic patterns in $O-C$ residuals to the radial velocity solution for the binary. While these patterns could have a connection to a magnetic cycle (e.g., through cycle modulated quadrupole moment changes; Applegate 1992; Lanza, Rodonò, \& 


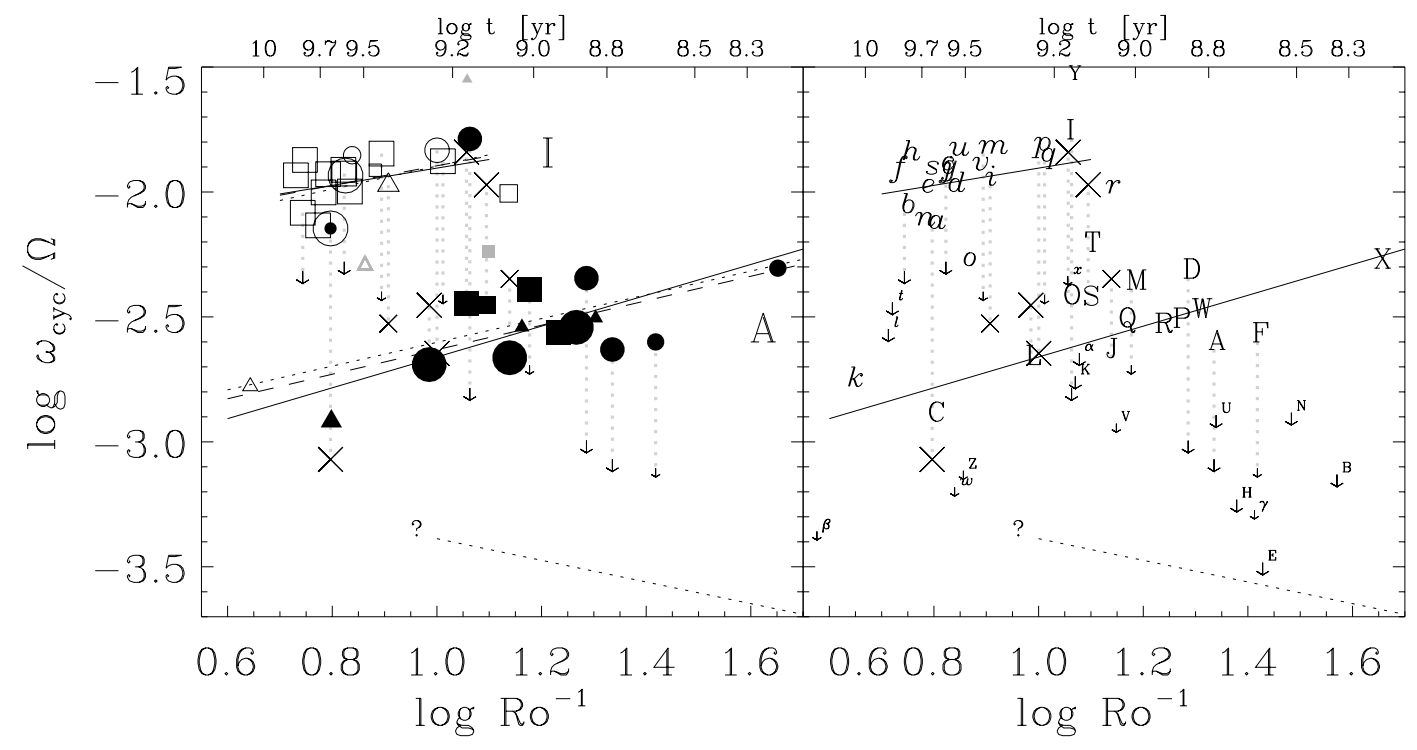

FIG. 1.-Left: $\omega_{\text {cyc }} / \Omega$ vs. $\operatorname{Ro}^{-1}$ (using $\tau_{\text {c, }, \mathrm{T}}$ ) for the expanded Mount Wilson sample (Table 1 ). The Sun, F, G, and $\mathrm{K}$ stars are indicated by dotted circle, open triangles, open circles, and open squares, respectively (filled if $\log \left\langle R_{\mathrm{HK}}^{\prime}\right\rangle \geq-4.75$, i.e., $\gtrsim$ the Vaughan-Preston 1980 gap; gray if not used in the fits). Symbol size $\propto \sqrt{w}$, a measure of the "reliability" of the $P_{\text {cyc }}$. Long-term trends (possible $P_{\text {cyc }}>25$ yr) are arrows, and larger arrows are trends noted by Bea95; only trends associated with a cycle are shown here. Dotted vertical lines connect stars with two $P_{\text {cyc }}$ (crosses mark $P_{\text {cyc }}^{(2)}$ ) or connect $P_{\text {cyc }}$ and a long-term trend). Least-squares fits $\left(\omega_{\text {cyc }} / \Omega \propto R_{0} \delta\right)$ for the active (A) and inactive (I) branches are shown: one weighted (by $\left.w\right)$ and including selected $P_{\text {cyc }}^{(2)}$ (solid; best-fit, $\delta$ values at top), one ignoring $P_{\text {cyc }}^{(2)}($ dashed; $1 w$ fit), and for comparison, fits from Paper I (dotted). The lower dashed line terminated with a "? " is a possible extension of the "superactive" (S) branch (see Fig. 5 and text). The approximate age $t$ (eq. [5]) is at top. See text for details and Table 3 for the fit parameters. Right: Same, but with all long-term trends included, and symbols from Table 1 identifying stars $\left(c, g\right.$, and $j$ overlap at log Ro $\left.^{-1} \approx 0.8\right)$.

Rosner 1998b), we viewed these $P_{\text {cyc }}$ with more caution, since they can also be caused by the presence of an undetected companion. As there is no equivalent of the FAP grade to judge the quality of the detections in most cases, we made a subjective assessment in assigning weights, $w$.

V833 Tau had the clearest cycle, given $w=4(C$; Hartmann et al. 1981; Bondar' 1995); PZ Mon's equally clear cycle (Bondar' 1995) could not be used ( $P_{\text {rot }}$ is unknown, and the star is of uncertain luminosity class; Saar 1998b).
Photometry of BY Dra $(B)$ and LQ Hya $(G)$ also showed reasonably clear cycles and were given $w=2$. We reanalyzed the photometry V471 Tau ( $F$; İbanoğlu et al. 1994) using the Scargle (1982) periodogram method and find two $P_{\text {cyc }}$ (the shorter matches periods in light level and $O-C$ residuals found by İbanoğlu et al.). We find a FAP $\approx 9 \times 10^{-3}$ for the main $P_{\text {cyc }}$, consistent with a "fair" $(w=2)$ grade. BY Dra also showed some evidence for 8 and $\approx 14 \mathrm{yr}$ cycles in photometry and/or $O-C$ residuals.

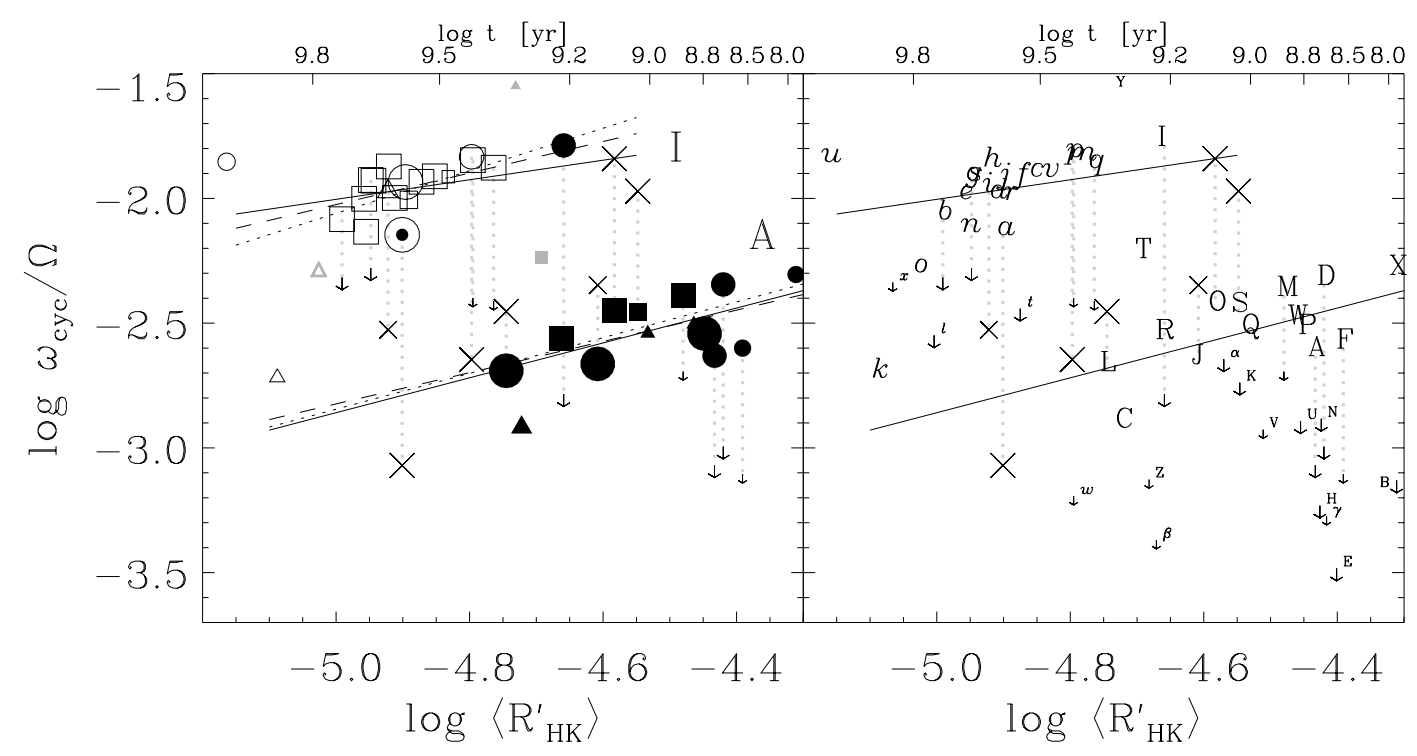

FIG. 2. - Left: $\omega_{\text {cyc }} / \Omega$ vs. $\left\langle R_{\mathrm{HK}}^{\prime}\right\rangle$ for the expanded Mount Wilson sample; symbols as in Fig. 1. Fits for $\omega_{\text {cyc }} / \Omega \propto\left\langle R_{\mathrm{HK}}^{\prime}\right\rangle^{v}$ are shown in the active (A) and inactive (I) branches for samples including selected $P_{\mathrm{cyc}}^{(2)}$ (solid; best-fit, $v$ at top), or excluding $P_{\mathrm{cyc}}^{(2)}$ (dashed; $1 w$ fit); the Paper I results are also shown (dotted). The approximate age (eq. [4]) is given at top. See text, Table 3 for details. Right: Same, but with all long-term trends included, and symbols from Table 1 identifying stars. 
TABLE 2

Stellar Properties for SuPeractive (S) Stars

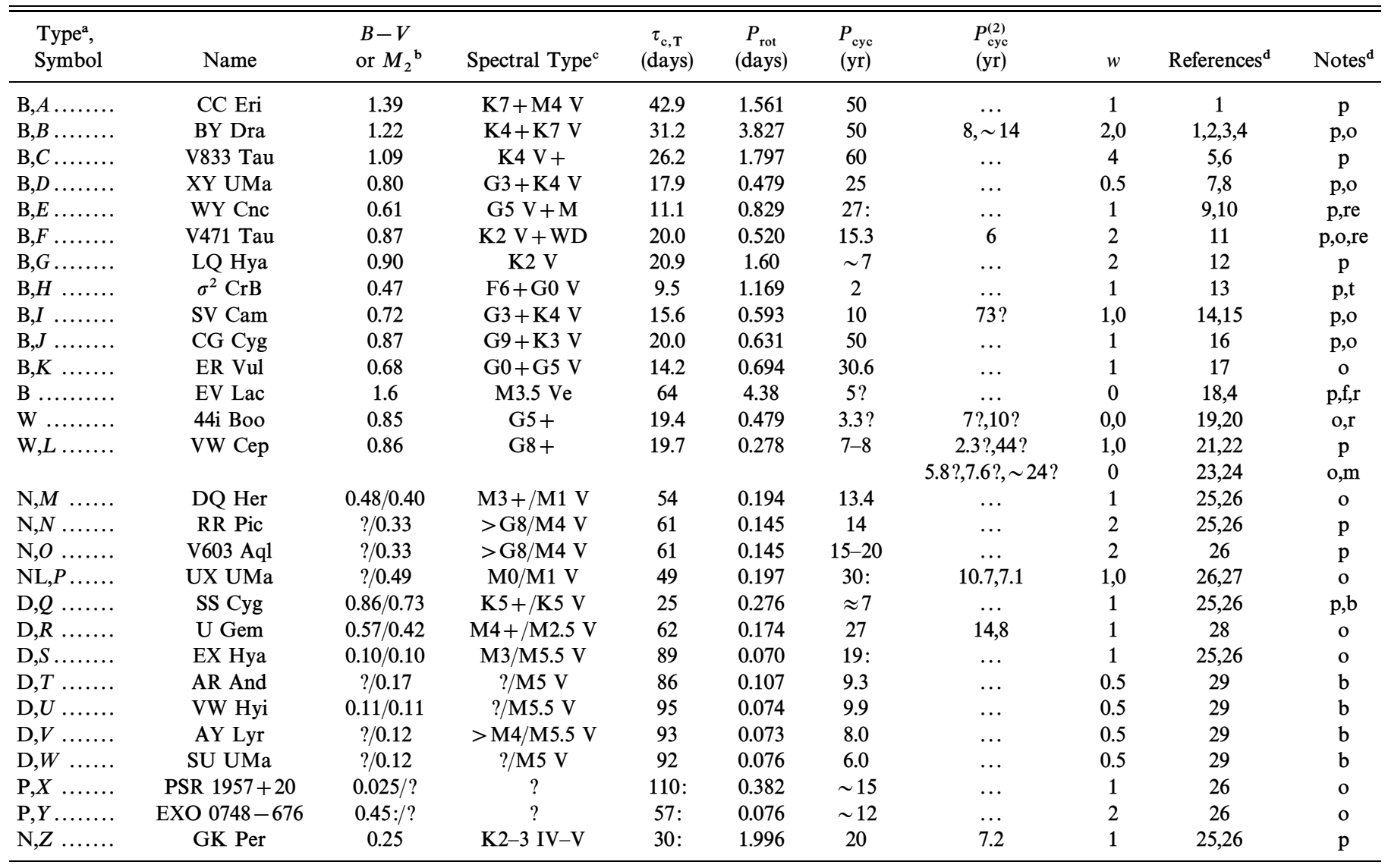

${ }^{\text {a }}$ Star type: $\mathrm{B}=\mathrm{BY}$ Dra, $\mathrm{W}=\mathrm{W}$ UMa, $\mathrm{D}=$ dwarf nova, $\mathrm{N}=$ nova, $\mathrm{NL}=$ nova-like, $\mathrm{P}=$ pulsar secondary.

${ }^{\text {b }}$ For CVs: a slash separates measured (Smith \& Dhillon 1998 or Patterson 1984) from estimated (from $P_{\text {rot }}$; Smith \& Dhillon 1998) values of $M_{2}$ (the secondary mass, in units of $\left.M_{\odot}\right)$. GK Per $M_{2}$ is from Reinsch 1994.

${ }^{c}$ For CVs: a slash separates spectroscopic (Patterson 1984) and $P_{\text {orb }}$-based estimates; GK Per spectral type is from Reinsch 1994.

d NoTES. - p: photometric $P_{\text {cyc }}$. o: $P_{\text {cyc }}$ from $O-C$ velocity residuals. b: $P_{\text {cyc }}$ from intervals between outbursts. m: $P_{\text {cyc }}$ from times of minimum. f: $P_{\text {cyc }}$ from numbers of flares. $\mathrm{t}: \tau_{c}$ based on $(B-V)_{2} \approx 0.58 \mathrm{r}$ : rejected (see text). re: reanalysis of published data.

REFERENCES.- (1) Phillips \& Hartmann 1978; (2) Rodonò \& Cutispoto 1992; (3) Mavridis et al. 1982; (4) Pettersen et al. 1992; (5) Hartmann et al. 1981; (6) Bondar' 1995; (7) Pojmanski \& Geyer 1990; (8) Henry et al. 1995; (9) Vivekananda Rao et al. 1991; (10) Heckert et al. 1998; (11) İbanoğlu et al. 1994; (12) Strassmeier et al. 1997; (13) Strassmeier et al. 1989; (14) Busso et al. 1985; (15) Frieboes-Conde \& Herczeg 1973; (16) Hall 1991b; (17) Shengbang, Qingyao, \& Yulan 1998; (18) Mavridis \& Avgoloupis 1986; (19) Liu et al. 1984; (20) Bergeat et al. 1972; (21) Bradstreet \& Guinan 1988; (22) Kaszás et al. 1998; (23) Bradstreet et al. 1997; (24) Karimie 1983; (25) Bianchini 1990; (26) Richman et al. 1994; (27) Rubenstein et al. 1991; (28) Smak 1993; (29) Bianchini 1992.

Less certain $(w=1)$ were CC Eri $\left(A\right.$; noisy variation), $\sigma^{2}$ $\mathrm{CrB}(H$; low amplitude, short time series; the Bakos $(1984)$ $P_{\text {cyc }}$ seems dubious), CG Cyg $(J$; poorly spaced data, though $P_{\text {cyc }}$ from both photometry and $O-C$ residuals roughly agree), SV Cam, WY Cnc, XY UMa, and ER Vul. In the case of SV Cam (I), the $P_{\text {cyc }}$ in $O-C$ had only a few data points and highly skewed shape (Busso, Scaltriti, \& Cellino 1985), while $P_{\text {cyc }}^{(2)}$ (Frieboes-Conde \& Herczeg 1973) may be due to a third component (Hall 1990). Combined photometry from two sources (Table 2 ) suggest a $P_{\text {cyc }} \approx 27$ yr for WY Cnc $(E)$, also quite uncertain. Despite inclusion in the Henry et al. (1995) tabulation, $P_{\text {cyc }}$ in XY UMa $(D)$ may be due to a third body (Pojmanski \& Geyer 1990); we reduced $w$. Older photometry and changes in flaring rates on EV Lac suggested $P_{\mathrm{cyc}} \approx 5 \mathrm{yr}$ (Mavridis \& Avgoloupis 1986), but later data show no cycle (Pettersen, Olah, \& Sandmann 1992); we neglect this star.

We also considered two W UMa systems based on Maceroni et al. (1989). The $10 \mathrm{yr}$ cycle of Bergeat et al. (1972) and the 3.3 yr cycle of Liu et al. (1984) for 44i Boo do not appear to hold up with more data and updated ephemeris solutions (cf. Burke et al. 1992 or Oprescu et al. 1996, which show no such $\left.P_{\text {cyc }}\right)$. VW Cep $(L)$ is a more complicated case, with $P_{\text {cyc }}=5.8$ (Bradstreet et al. 1997), 7.6, and $21.3 \mathrm{yr}$ (Kaszás et al. 1998) seen in $O-C$ residuals, $P_{\text {cyc }}=9.5$ and $25.9 \mathrm{yr}$ in time-of-minimum differences (Kaszás et al. 1998), and $P_{\mathrm{cyc}} \approx 7-8 \mathrm{yr}$ (Bradstreet \& Guinan 1988), 2.3 and $44 \mathrm{yr}$ (Karimie 1983) attested to photometrically. We take $P_{\text {cyc }} \sim$ $7.5 \mathrm{yr}$ (combining $O-C$ and photometric periods) as the primary period.

Most stars in this section are close binaries where tidal effects are potentially important for the dynamo. Thus, we caution that their dynamo behavior may not be strictly comparable to that of single stars.

\subsection{The Secondaries of Cataclysmic Variables}

Photometry of some cataclysmic variables (CVs) between accretion outbursts show quasi-cyclic behavior on timescales of years, and still others show quasi-periodic changes in orbital period $P_{\text {orb }}$, or in outbursts intervals (Bianchini 1990, 1992). Some researchers (Bianchini 1987, 1990; Warner 1988) believe these variations are caused by a mag- 
netic starspot cycle on the system's low-mass secondary star (see Hall 1990 and Bianchini 1992 for further discussion). As the secondaries rotate very rapidly $\left(P_{\text {rot }}=P_{\text {orb }}\right.$ is of order hours), high magnetic activity levels are expected and, indeed, are observed in a few cases (e.g., Balmer emission; Robinson et al. 1981, Dhillon, Jones, \& Marsh 1994). Magnetic fields on CV secondaries have long been invoked to help explain elements of CV evolution, especially the wellknown "period gap" (e.g., Verbunt \& Zwaan 1981; Tout \& Pringle 1992; Kolb, King, \& Ritter 1998). Theory of dynamos in CV secondaries has been investigated by many groups (recently, e.g., by Zangrilli, Tout, \& Bianchini 1997). Preliminary comparison of cycles on late-type stars and CVs shows both groups have similar distributions of $P_{\mathrm{cyc}}$ (Maceroni et al. 1989).

Bianchini $(1990,1992)$ compiled a list of cycle data on CV secondaries. A critical reanalysis by Richman, Applegate, \& Patterson (1994) found most of the variations are not strictly periodic or were otherwise poorly defined or uncertain. They offered a few additional cycle candidates (including two pulsar systems) and, despite few clear $P_{\text {cyc }}$ measurements, ultimately ruled in favor of the existence of dynamo cycles in CV secondaries. We follow the Richman et al. analysis and discussion in selecting the best candidate cycles, rejecting systems with unknown $P_{\text {rot }}$ and giving GK $\operatorname{Per}(Z)$ lower weight (evolved, less certain $\tau_{c}$ ). Several additional stars listed in Bianchini (1992) but not studied by Richman et al. were included but were given lower $w$ (e.g., $P_{\text {cyc }}$ based on intervals between superoutbursts in SU UMa objects, which are not connected to magnetic cycles by any theory; Bianchini 1992). For U Gem $(R)$, we take the three $P_{\text {cyc }}$ Smak (1993) derived formally, rather than his general conclusion that $P_{\text {cyc }} \sim 10$ yr. Of the remaining stars, SS Cyg $(Q)$ and $\mathrm{U}$ Gem are more uncertain because of possible multiple $P_{\text {cyc }}$, and the periodicity of UX UMa $(P)$ " may not be strict" (Rubenstein, Patterson, \& Africano 1991). There is also the puzzle of apparent cycles on stars that should be completely convective $\left(M_{2} / M_{\odot} \lesssim 0.3\right)$ and thus not be capable of cyclic dynamos (e.g., Rädler 1986); these are given lower weight. Again, where possible we have assessed the $P_{\text {cyc }}$ quality by examining the time series; our final list consists of 14 stars (Table 2).

As in BY Dra systems, tidal synchronization of $P_{\text {rot }}$ with the orbit will likely alter the dynamo properties at some level. Some caution is advised in comparing the cycle results directly with single stars. One must also be wary of the possibility that a third component could mimic a $P_{\text {cyc }}$ based on $O-C$ data (e.g., Wolf et al. 1993) or low-level, periodic mass transfer and subsequent heating (e.g., King et al. 1996), could mimic a photometric cycle.

Finally, we add a cautionary note: cycle-like behavior can be mimicked by multiple active areas with finite lifetimes on a differentially rotating star (Eaton, Henry, \& Fekel 1996). Also, the purely visual identification of cycles can be risky (Press 1978). Longer time series and careful analysis of variability timescales (e.g., Donahue et al. 1997) are needed to help exclude false cycle signals.

\subsection{Adopted Stellar Parameters}

Basic stellar data (spectral type, $B-V, P_{\text {rot }}$ ) came from a variety of sources. For the Mount Wilson stars (§ 2.1), $\left\langle R_{\mathrm{HK}}^{\prime}\right\rangle$ were taken from Bea95, while spectral types and $B-V$ came from Rea98 or Bea95; however, we modified the luminosity classes in some cases (see below). Values of
$T_{\text {eff }}$ were estimated from $\log T_{\text {eff }}=3.908-0.234(B-V)$ for $B-V \leq 1.4$ (Nea84; in good agreement with, e.g., Alonso, Arribas, \& Martinez-Roger 1996). For redder stars, we used the $\log T_{\text {eff }}$-spectral type-color relations in Bessell (1991), modified slightly to reflect the $T_{\text {eff }}$ estimates of Leggett et al. (1996). We took $P_{\text {rot }}$ values (as in Paper I) from Donahue et al. (1996, 1997), or Baliunas, Sokoloff, \& Soon (1996b), in order of preference (except HD 3651, taken from Baliunas et al.). Basic data on the active spotted stars (\$ 2.2) was drawn from Strassmeier et al. $(1993,1997)$ and references in Table 2. $P_{\text {rot }}$ for the CV systems ( $\left.\$ 2.3\right)$ are mostly from Bianchini (1990).

We explored several methods to compute $\tau_{c}$. Nea84 give a formula for an empirical $\tau_{\mathrm{c}, \mathrm{E}}$ that best fitted the $\left\langle\boldsymbol{R}_{\mathrm{HK}}^{\prime}\right\rangle-$ $\mathrm{Ro}^{-1}$ relationship for data then available; this was the value used in Paper I. Saar (1998a) derived a revised $\tau_{\mathrm{c}, \mathrm{E}}$ by a similar method based on the latest $\left\langle R_{\mathrm{HK}}^{\prime}\right\rangle$ and $P_{\text {rot }}$ (see above) but giving less precise $P_{\text {rot }}$ (Baliunas et al. 1996b) half-weight and excluding $\mathscr{F}$ stars. The latter were rejected because they may be in magnetic grand minima (Baliunas \& Jastrow 1990); retaining them would bias the fit, since they nearly all show $\left\langle R_{\mathrm{HK}}^{\prime}\right\rangle$ values below other stars with the same $B-V$ and $P_{\text {rot. A quartic fit then }}$ yielded $\tau_{\mathrm{c}, \mathrm{E}}=-3.3300+15.382(B-V)-20.063(B-V)^{2}$ $+12.540(B-V)^{3}-3.1466(B-V)^{4}$ for $B-V \leq 1.0$ (Fig. 3). For $B-V>1.0$, we set $\tau_{c, \mathrm{~B}}=25$ days (cf. Stepień 1989), since the drop in $\tau_{\mathrm{c}, \mathrm{E}}$ likely does not reflect the actual $\tau_{c}$ (see below). The new $\tau_{\mathrm{c}, \mathrm{E}}$ values differ from those of Nea84 primarily in the most massive $(B-V \leq 0.50)$ and least massive $(B-V \geq 0.95)$ stars (Fig. 3).

While empirical $\tau_{\mathrm{c}, \mathrm{E}}$ work well in $\mathrm{Ro}^{-1}$-activity correlations (as designed!), they differ from theoretical $\tau_{\mathrm{c}, \mathrm{T}}$ values at the extremes in mass and gravity. This may be because $\tau_{\mathrm{c}, \mathrm{E}}$ combines two effects: the "true" turnover time $\left(\sim \tau_{\mathrm{c}, \mathrm{T}}\right)$ related to magnetic field production, and some function describing the efficiency and means of the conversion of magnetic energy into atmospheric heating. Thus, $\tau_{\mathrm{c}, \mathrm{T}} \mathrm{might}$ be more appropriate to connect dynamo parameters (see also Ossendrijver 1997). In addition, Gunn, Mitrou, \& Doyle (1998, hereafter GMD) find that $\tau_{\mathrm{c}, \mathrm{T}}$ is slightly pre-

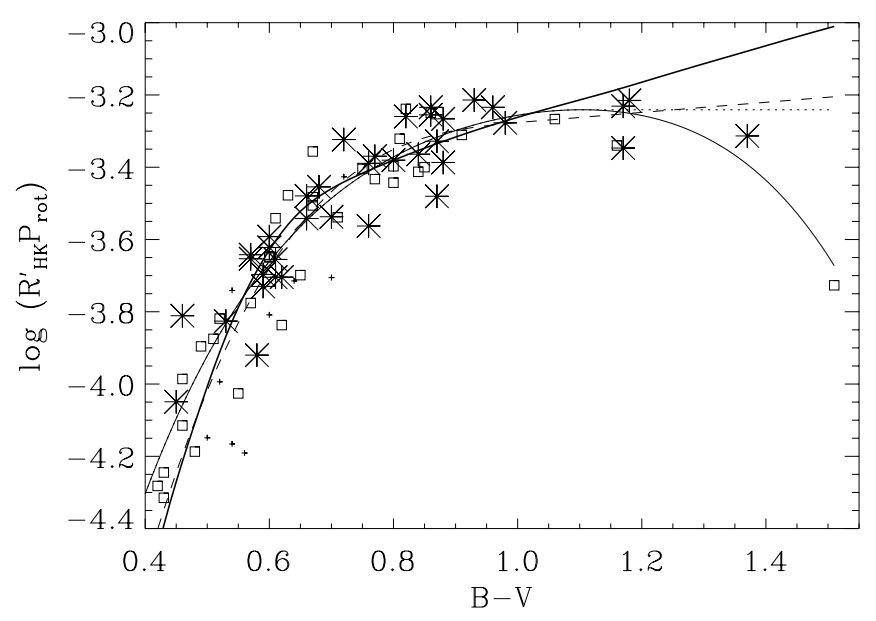

FIG. 3.-Plot of $R_{\mathrm{HK}}^{\prime} P_{\text {rot }}$ vs. $B-V$ showing stars with well-determined $P_{\text {rot }}$ (asterisks; Donahue et al. 1996, 1997) and less precise $P_{\text {rot }}$ (flat activity stars, plus signs; others, open squares; Baliunas et al. 1996b). Curves of various $\tau_{c}$ are shown: $\tau_{\mathrm{c}, \mathrm{E}}$ of Noyes et al. (1984b; dashed), a modified theoretical $\tau_{\mathrm{c}, \mathrm{T}}$ (Gunn et al. 1998; thick solid), and the new $\tau_{\mathrm{c}, \mathrm{E}}$ calibration of Saar (1998a; fourth-order fit, solid; extension for $B-V>1.1$, dotted). Note the flat stars mostly lie below the fits. 
ferred over $\tau_{\mathrm{c}, \mathrm{E}}$ in rotation-activity relations for evolved binaries.

To compute $\tau_{\mathrm{c}, \mathrm{T}}$ for dwarfs, we interpolated values from GMD (their ZAMS models), linearly extrapolating (in $\left.\log \tau_{\mathrm{c}, \mathrm{T}}\right)$ for $\log T_{\text {eff }}<3.60$. The resulting $\tau_{\mathrm{c}, \mathrm{T}}$ values were scaled slightly to best match the data in Figure 3. For evolved stars, $\tau_{c}$ is a sensitive function of mass and age, making estimates less precise. In these cases, we combined Hipparcos parallaxes ( $\pi$; ESA 1997) and bolometric corrections of Flower (1996; corrected by +0.028 to match the Sun) to estimate $M_{V}$ and luminosities. The luminosities were used with $T_{\text {eff }}$ to estimate masses and thence evolutionary corrections to $\tau_{\mathrm{c}, \mathrm{T}}$ using the models of GMD. We also used $M_{V}$ and Figure 1 of Rea98 to revise luminosity classes where needed.

In the spotted dwarfs ( $(2.2)$, computing $\tau_{c}$ from $B-V$ weights the result toward the primary star, where contribution to the total variability is typically greatest. However, since F stars have small photometric amplitudes (e.g., Lockwood, Skiff, \& Radick 1997), for $\sigma^{2} \mathrm{CrB}(H)$ we assumed the $\mathrm{G}$ dwarf secondary is responsible for the variation and used its estimated $B-V$ to compute $\tau_{c}$.

Since colors of $\mathrm{CV}$ systems are complicated by the primary star and the accretion disk, we used masses and/or spectral types of the secondaries from Patterson (1984) and Smith \& Dhillon (1998) to estimate their $\tau_{c}$ values. When neither was available, we used $P_{\text {rot }}$ to estimate the secondary mass, $M_{2}$ and the spectral type (Smith \& Dhillon 1998). The final $\tau_{c}$ (Table 2) was the average of those computed from all available means (we use only $\tau_{\mathrm{c}, \mathrm{T}}$, as $\tau_{\mathrm{c}, \mathrm{E}}$ is not well calibrated for M dwarfs). We caution that these $\tau_{c}$ values are uncertain; the various estimates sometimes disagreed, and since the secondary has enhanced, episodic mass loss, its internal structure may differ from a single star of the same mass.

\section{RESULTS}

\subsection{The "Active" $(A)$ and "Inactive" $(I)$ Branches}

Combining the "reliable" cycles $(w>0)$ and trends in Table 1 and constructing correlations between $\omega_{\text {cyc }} / \Omega$ and $\mathrm{Ro}^{-1}$ or $\left\langle R_{\mathrm{HK}}^{\prime}\right\rangle$ leads to Figures 1 and 2 . These include 36 $P_{\text {cyc }}$ (14 graded excellent, six graded good, 11 graded fair, and five new/ungraded), seven $P_{\text {cyc }}^{(2)}$ (two good, four fair, one new), and 21 long-term trends (five new). Here we have scaled the size of the symbols to the reliability $(w)$ of the $P_{\mathrm{cyc}}$ value, or whether the trend was identified in Bea95 (larger arrows).

We first focus on how the added Wilson survey stars affect the results of Paper I. Figures 1 and 2 show the correlations between $\omega_{\text {cyc }} / \Omega$ and $\mathrm{Ro}^{-1}$ or $\left\langle R_{\mathrm{HK}}^{\prime}\right\rangle$. One immediately sees a division into two roughly parallel branches, separated by a factor of $\approx 6$ in $\omega_{\text {cyc }} / \Omega$, which we designate inactive (I; upper branch) and active (A; lower branch). In Paper I, the branches were strictly segregated by activity: $\log \left\langle R_{\mathrm{HK}}^{\prime}\right\rangle \approx-4.75$ divided I and A branch stars, and all stars belonged to a branch. Here, there are exceptions to these clean divisions: HD 76151 (I) lies on the I branch and HD $136202(k)$ on the A branch, and HD 190007 (T) and HD $187691(o)$ fall between the branches, as do the $P_{\mathrm{cyc}}^{(2)}$ of HD $78366(\mathrm{~J})$ and HD $114710(\mathrm{~L})$.

We computed many types of least-squares fits: unweighted and weighted (by $w$ ), different $\tau_{c}$, and including or ignoring some or all of the $P_{\mathrm{cyc}}^{(2)}$ and/or evolved stars. The $w$ values in the weighted fits were renormalized such that $\Sigma_{1}^{n} w=n$, so that the standard deviations of the fits, $\sigma_{\mathrm{fit}}$ in the weighted and unweighted cases could be more directly compared. A representative selection of the results for the many possible fits are given in Table 3 and Figures 1 and 2; fits from Paper I are also given.

We believe the weighted fits including selected $P_{\text {cyc }}^{(2)}$ (the " $2 w$ " fits) have the most complete and reliable stellar sample. These fits include all $P_{\text {cyc }}$ with $w>0$ (except stars T and $o$ ) and all $P_{\text {cyc }}^{(2)}$, except two stars whose secondary periods fail to fully "jump" the I-A gap (stars J and L). The other main categories of fits are those ( $3 w$ fits) including all $P_{\mathrm{cyc}}^{(2)}$ (with $P_{\mathrm{cyc}}^{(2)}$ for stars $\mathrm{J}$ and $\mathrm{L}$ placed on the $\mathrm{A}$ branch), those excluding all $P_{\mathrm{cyc}}^{(2)}(1 w$ fits $)$, and those excluding evolved (IV-V, IV) stars ( $5 w$ fits). Typically we use our modified $\tau_{\mathrm{c}, \mathrm{T}}$ throughout (see $\S 2.4$ ), but we also list a representative fit for $\mathrm{Ro}^{-1}$ using $\tau_{\mathrm{c}, \mathrm{E}}(4 w$ fit $)$.

The fits to the branches are generally similar to those in Paper I; the largest change is in the I branch $\left\langle R_{\mathrm{HK}}^{\prime}\right\rangle$ fit (Fig. 2 ). The addition of the new stars makes the standard deviation of the fit, $\sigma_{\mathrm{fit}}$, slightly smaller for the I branch, and

TABLE 3

FITS $\left(\omega_{\mathrm{cyc}} / \Omega=c x^{b}\right)$ AND DeRIVEd PARAMETERS

\begin{tabular}{|c|c|c|c|c|c|}
\hline$x$ Var. & Fit Type ${ }^{a}$ & $n$ & $\log c$ & $b$ & $\begin{array}{c}\sigma_{\mathrm{fit}} \\
(\mathrm{dex})\end{array}$ \\
\hline$\left\langle R_{\mathrm{HK}}^{\prime}\right\rangle \ldots \ldots$ & $\mathrm{I} 0^{\mathrm{b}}$ & 14 & 2.204 & 0.853 & 0.109 \\
\hline$\left\langle R_{\mathrm{HK}}^{\prime}\right\rangle \ldots \ldots$ & $\mathrm{A} 0^{\mathrm{b}}$ & 6 & 0.739 & 0.717 & 0.097 \\
\hline $\mathrm{Ro}^{-1}$. & $\mathrm{I} 0^{\mathrm{b}}$ & 14 & -2.355 & 0.458 & 0.097 \\
\hline $\mathrm{Ro}^{-1}$. & $\mathrm{A} 0^{\mathrm{b}}$ & 6 & -3.077 & 0.475 & 0.109 \\
\hline$\Omega \ldots$. & $\mathrm{I} 0+\mathrm{A} 0^{\mathrm{b}}$ & 20 & -3.900 & -1.198 & 0.128 \\
\hline $\mathrm{Ro}^{-1}$ & $\mathrm{I} 1 w^{\mathrm{b}}$ & 19 & -2.289 & 0.393 & 0.0933 \\
\hline $\mathrm{Ro}^{-1}$. & $\mathrm{I} 2 w^{\mathrm{c}}$ & 21 & -2.249 & 0.345 & 0.0916 \\
\hline $\mathrm{Ro}^{-1}$. & $\mathrm{I} 2$ & 21 & -2.199 & 0.291 & 0.0913 \\
\hline $\mathrm{Ro}^{-1}$. & $\mathrm{I} 4 w$ & 21 & -2.358 & 0.476 & 0.0914 \\
\hline $\mathrm{Ro}^{-1}$. & $\mathrm{I} 5 \mathrm{w}$ & 20 & -2.253 & 0.347 & 0.0907 \\
\hline $\mathrm{Ro}^{-1}$. & $\mathrm{A} 1 w^{\mathrm{b}}$ & 15 & -3.122 & 0.490 & 0.123 \\
\hline $\mathrm{Ro}^{-1}$. & $\mathrm{A} 2 w^{\mathrm{c}}$ & 18 & -3.277 & 0.617 & 0.144 \\
\hline $\mathrm{Ro}^{-1}$. & $\mathrm{A} 2$ & 18 & -3.202 & 0.544 & 0.143 \\
\hline $\mathrm{Ro}^{-1}$. & $\mathrm{A} 3 w$ & 20 & -3.189 & 0.556 & 0.154 \\
\hline $\mathrm{Ro}^{-1}$. & $\mathrm{A} 4 w$ & 18 & -3.265 & 0.606 & 0.159 \\
\hline $\mathrm{Ro}^{-1}$. & $\mathrm{A} 5 w$ & 16 & -3.227 & 0.577 & 0.146 \\
\hline $\mathrm{Ro}^{-1}$. & $\mathrm{S} 1 w^{\mathrm{b}}$ & 26 & -2.545 & -0.546 & 0.309 \\
\hline $\mathrm{Ro}^{-1}$. & $S 6 w^{\mathrm{c}}$ & 24 & -2.954 & -0.433 & 0.220 \\
\hline $\mathrm{Ro}^{-1}$ & S6 & 24 & -2.983 & -0.415 & 0.217 \\
\hline$\left\langle R_{\mathrm{HK}}^{\prime}\right\rangle \ldots \ldots$ & $\mathrm{I} 1 w^{\mathrm{b}}$ & 19 & 1.134 & 0.632 & 0.0977 \\
\hline$\left\langle R_{\mathrm{HK}}^{\prime}\right\rangle \ldots \ldots$ & $\mathrm{I} 2 w^{\mathrm{c}}$ & 21 & -0.045 & 0.392 & 0.0954 \\
\hline$\left\langle R_{\mathrm{HK}}^{\prime}\right\rangle \ldots \ldots$ & $\mathrm{I} 2$ & 21 & -0.771 & 0.241 & 0.0925 \\
\hline$\left\langle R_{\mathrm{HK}}^{\prime}\right\rangle \ldots \ldots$ & $\mathrm{I} 5 w$ & 20 & 0.372 & 0.478 & 0.0842 \\
\hline$\left\langle R_{\mathrm{HK}}^{\prime}\right\rangle \ldots \ldots$ & $\mathrm{A} 1 w^{\mathrm{b}}$ & 15 & 0.308 & 0.626 & 0.129 \\
\hline$\left\langle R_{\mathrm{HK}}^{\prime}\right\rangle \ldots \ldots$ & $\mathrm{A} 2 w^{\mathrm{c}}$ & 18 & 0.637 & 0.699 & 0.156 \\
\hline$\left\langle R_{\mathrm{HK}}^{\prime}\right\rangle \ldots$. & $\mathrm{A} 2$ & 18 & 0.003 & 0.561 & 0.153 \\
\hline$\left\langle R_{\mathrm{HK}}^{\prime}\right\rangle \ldots \ldots$ & $\mathrm{A} 3 w$ & 18 & 0.423 & 0.649 & 0.162 \\
\hline$\left\langle R_{\mathrm{HK}}^{\prime}\right\rangle \ldots \ldots$ & $\mathrm{A} 5 w$ & 16 & 0.818 & 0.737 & 0.143 \\
\hline$\Omega \ldots \ldots \ldots$ & $\mathrm{I} 1 w+\mathbf{A} 1 w+\mathrm{S} 6 w$ & 58 & -2.844 & -1.098 & 0.306 \\
\hline$\Omega \ldots \ldots \ldots$ & $\mathrm{I} 2 w+\mathbf{A} 2 w+\mathrm{S} 6 w^{\mathrm{c}}$ & 63 & -2.848 & -1.094 & 0.324 \\
\hline $\mathrm{Ro}^{-1}$. & comb- $2 w$ & 63 & $\ldots$ & $\ldots$ & 0.138 \\
\hline
\end{tabular}

${ }^{a}$ I, A, S: inactive, active, superactive branch fit; 0 : from Paper I (using $\tau_{\mathrm{c}, \mathrm{E}}$ ). 1: Expanded sample (using $\tau_{\mathrm{c}, \mathrm{T}}$ ). 2: Expanded sample (using $\tau_{\mathrm{c}, \mathrm{T}}$ ) + selected $P_{\mathrm{cyc}}^{(2)}$. 3: Expanded sample (using $\tau_{\mathrm{c}, \mathrm{T}}$ ) + all $P_{\mathrm{cyc}}^{(2)}$. 4: Expanded sample (using $\tau_{\mathrm{c}, \mathrm{T}}$ ) + selected $P_{\mathrm{cyc}}^{(2)}$ using $\tau_{\mathrm{c}, \mathrm{E}}$. 5: Expanded sample (using $\left.\tau_{\mathrm{c}, \mathrm{T}}\right)+$ selected $P_{\mathrm{cyc}}^{(2)}-$ evolved stars. 6: Expanded sample (using $\tau_{\mathrm{c}, \mathrm{T}}$ ) - "transition branch stars" (see text). $w$ : weighted. comb-2w: $\mathrm{I} 2 w+\mathrm{A} 2 w+\mathrm{S} 6 w$ (combined fit, 6 degrees of freedom)

${ }^{b}$ Plotted in Figs. 1, 2, and/or 5.

c Plotted in Figs. 1, 2, and/or 5, best fit. 


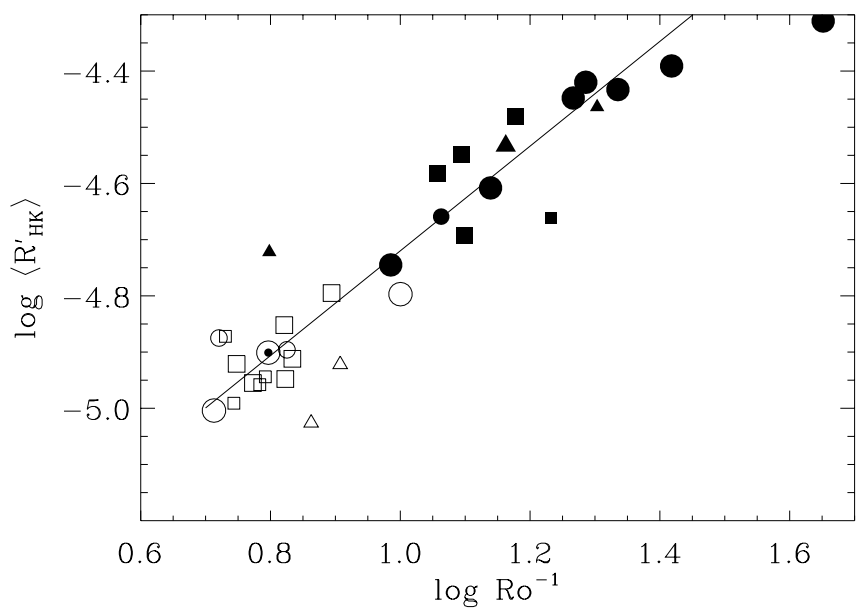

FIG. 4. $-\left\langle R_{\mathrm{HK}}^{\prime}\right\rangle$ vs. $\mathrm{Ro}^{-1}$ (using $\tau_{\mathrm{c}, \mathrm{T}}$ ) for the expanded Mount Wilson sample with measured $P_{\text {cyc }}$ and $P_{\text {rot }}$; symbols as in Fig. 2. A weighted (like Fig. 3) fit excluding saturated stars with $\log \left\langle R_{\mathrm{HK}}^{\prime}\right\rangle \geq-4.4$ and star $r$, yields $\left\langle R_{\mathrm{HK}}^{\prime}\right\rangle \propto \mathrm{Ro}^{-0.93}$ with $\sigma_{\mathrm{fit}}=0.077$ (31 stars). The fit improves if evolved stars (with uncertain $\tau_{c}$ ) are neglected $\left(\sigma_{\mathrm{fit}}=0.063\right)$.

larger for the A branch (which now has $\sim 3$ times more stars). A few individual stars can notably change $\sigma_{\text {fit }}$ : the subgiant HD $161239(u)$, for example, despite a low $w$, wields leverage on the fit in Figure 2 because of its tiny $R_{\mathrm{HK}}^{\prime}$ (cf. the I $2 w$ and I $5 w$ fits). Gross changes in the stellar sample can also occasionally alter the results (e.g., eliminating all $P_{\mathrm{cyc}}^{(2)}$ in the $1 w$ I-branch HK fit). For the most part, though, the relations seem fairly stable to changes in weighting and star selection (Table 3).

Since $\left\langle R_{\mathrm{HK}}^{\prime}\right\rangle \propto \mathrm{Ro}^{\mu}$, where $\mu \approx 1.0$ (Paper I; Fig. 4), the slopes of the fits between $\omega_{\text {cyc }} / \Omega$ and $\mathrm{Ro}^{-1}$ or $\left\langle R_{\mathrm{HK}}^{\prime}\right\rangle$ should be similar for a given branch (i.e., $\mu v \approx \delta$ ). In Paper I, this was not the case: because of the limited number of stars (A branch), and the limited range of observed variables (I branch), the slopes did not agree well (I0, A0 fits; Table 3). For the choices of stars and $w$ explored, we found $\omega_{\text {cyc }} / \Omega \propto$ $\mathrm{Ro}^{-\delta}$ with $0.29 \leq \delta_{I} \leq 0.48$ and $0.44 \leq \delta_{A} \leq 0.62$. Similar fits for $\omega_{\text {cyc }} / \Omega \propto\left\langle R_{\mathrm{HK}}^{\prime}\right\rangle^{v}$ yielded $0.24 \leq v_{I} \leq 0.85$ and $0.52 \leq v_{A} \leq 0.74$. In our most favored case (the $2 w$ fits) and for several other fits, the Ro and $\left\langle R_{\mathrm{HK}}^{\prime}\right\rangle$ fits are very similar on each branch (Table 3), thus yielding the branch fits that are more internally consistent than in Paper I. The branches are separated by a factor of $\approx 6.1$ in the $\mathrm{Ro}^{-1}$ relation (for $\left.0.7 \leq \log \mathrm{Ro}^{-1} \leq 1.1\right)$ and a factor of $\approx 6.3$ in the $\left\langle R_{\mathrm{HK}}^{\prime}\right\rangle$ relation (for $-5.10 \leq \log \left\langle R_{\mathrm{HK}}^{\prime}\right\rangle \leq-4.55$ ), yielding an average separation of a factor of $\approx 6.2$, as in Paper I.

\subsection{The "Superactive" (S) Branch and Extensions}

When we also add the stars from Table 2, we find the plot of $\omega_{\text {cyc }} / \Omega$ versus $\mathrm{Ro}^{-1}$ is considerably extended (Fig. 5). Indeed, the additional stars appear to form a new (though somewhat more poorly defined) branch that, unlike the A and I branches, has $\omega_{\text {cyc }} / \Omega$ decreasing with increasing $\mathrm{Ro}^{-1}$. We call this the "superactive" (S) branch. If we fit these (though here ignoring the less certain $P_{\mathrm{cyc}}^{(2)}$ ) using the same procedure as above, we find $\omega_{\text {cyc }} / \Omega \propto \mathrm{Ro}^{0.55}$ (S1 $w$ fit; Table 3 ). The standard deviation of the fit can be reduced considerably (from $\sigma_{\text {fit }}=0.31$ dex to $0.22 \mathrm{dex}$ ), however, if two anomalous stars (LQ Hya $=G, \sigma^{2} \mathrm{CrB}=H$ ) are excluded. In this case, a weighted fit (S6w) yields $\omega_{\text {cyc }} / \Omega \propto \mathrm{Ro}^{0.43}$. We adopt this fit, for reasons detailed below, as the "best" one.

If the "anomalous stars" do not belong on the S branch, their position in the $\omega_{\text {cyc }} / \Omega$ versus $\mathrm{Ro}^{-1}$ plane suggests they may be transitional between the $\mathrm{S}$ and $\mathrm{A}$ branches. Consistent with this, another active spotted star (star X; § 2.1) lies on the high $\mathrm{Ro}^{-1}$ end of the A branch. To further explore this idea, we searched for other active stars in this $\mathrm{Ro}^{-1}$ range; many RS CVns are suitable, but since they are sig-

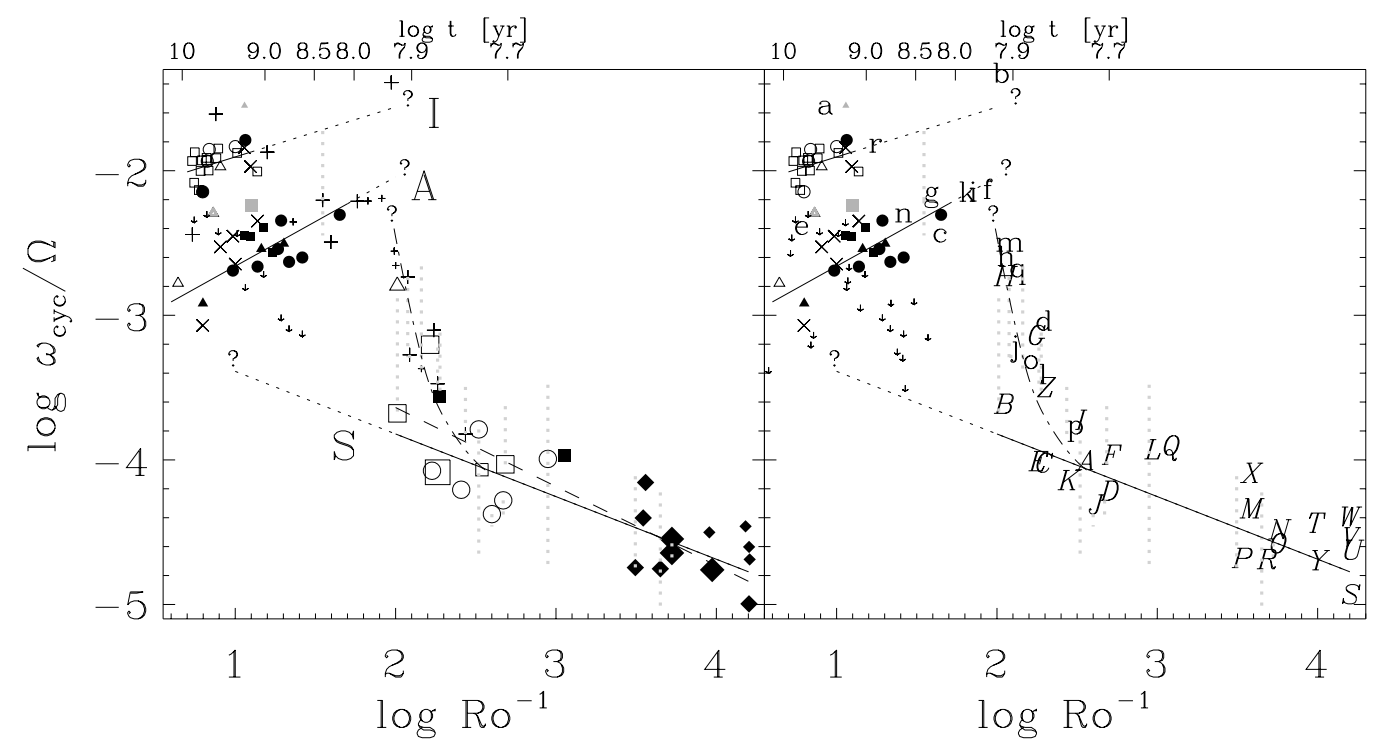

FIG. 5.-Left: $\omega_{\text {cyc }} / \Omega$ vs. Ro ${ }^{-1}$ for all stars, showing a "superactive" (S) branch (Fig. 2 expands the upper left-hand corner of this plot). Symbols as in Fig. 2 ; in addition, M dwarfs (diamonds), RS CVn's (plus signs), and (for log Ro ${ }^{-1} \gtrsim 2$ ) spotted dwarfs (open symbols; $§ 2.2$ ) and CV secondaries ( filled symbols; $\S$ 2.3) are indicated. Here, for clarity, dashed lines connect various $P_{\text {cyc }}$, and symbol sizes are $\propto \sqrt{w}$ only for stars not already in Fig. 2. Weighted fits are shown to $\omega_{\text {cyc }} / \Omega \propto \mathrm{Ro}^{-\delta_{\mathrm{s}}}$ including (dashed; S1w fit) or ignoring (solid, best fit, $\delta$ at top) “anomalous" stars $G$ and $H$; possible extensions to the latter, and to the I and A branches, are also drawn (dotted). The steep dash-dotted curve depicts a possible transition zone between the $\mathrm{S}$ and $\mathrm{A}$ branches that is consistent with many RS CVn's and may explain the "anomalous" stars. The approximate "rotational" age $t$ (less accurate for log $t \lesssim 8.0$, and differing from evolutionary age in evolved stars and close binaries) is at top (from eq. [5], modified). See text and Table 4 for details. Right: Same, but with all long-term trends shown, and symbols from Tables 2 and 4 identifying stars not in Fig. 1. 
nificantly evolved, $\tau_{c}$ is less certain. We have compiled a list of $P_{\text {cyc }}$ in active evolved systems (RS CVn binaries and one pre-MS star; Table 4). We either took $\tau_{\mathrm{c}, \mathrm{T}}$ directly from GMD or used their models to estimate it, computing $\tau_{\mathrm{c}, \mathbf{T}}$ for the star(s) in the RS CVn system likely responsible for the cycle (Table 4).

When the active evolved stars are added (Fig. 5, plus signs), eight seem to delineate (with the "anomalous" dwarfs) a sharp transition region between the S and A branch (Fig. 5, steep dotted curve). A further three RS CVns ( $\mathrm{f}, \mathrm{i}$, and $\mathrm{k}$ ) appear to occupy a high $\mathrm{Ro}^{-1}$ extension of the $\mathrm{A}$ branch (Fig. 5, dotted), three more lie on the A branch (c, g, and $\mathrm{n}$ ), and two (b and r) occupy a high $\mathrm{Ro}^{-1}$ extension of the I branch (Fig. 5, dotted). Two stars (a and e) are not clearly associated with a branch, but these have less certain $\tau_{c}$. Thus, despite the uncertain $\tau_{\mathrm{c}, \mathbf{T}}$ values, nearly all the stars appear to either lie on extensions of the I or A branch, or support the idea of a steep S-to-A branch transitional regime. The $P_{\mathrm{cyc}}^{(2)}$ for BY Dra $(B)$ may also be consistent with this transitional phase.

\section{DISCUSSION}

Several general comments can be made. First, careful addition of "fair" graded cycles from the Mount Wilson program seems to verify and extend relationships found for a smaller group of higher graded $P_{\text {cyc }}$ in Paper I. Despite the addition here of many less certain cycles, only two of the 36 stars (T and $o$ ) do not have a primary $P_{\text {cyc }}$ clearly associated with one branch or the other. The record for $P_{\mathrm{cyc}}^{(2)}$ is less clear. If we require that the two cycles are in opposite branches ( 2 and $2 w$ fits), two (stars $\mathbf{J}$ and L) of seven stars fail the test; if this requirement is relaxed, all seven are close to a branch ( $3 w$ fits).

Long-term trends of I-branch stars, with only one exception (star I), all have upper limits to $\omega_{\text {cyc }} / \Omega$ well above the A branch. The existence of a $P_{\mathrm{cyc}}^{(2)}$ in the Sun (Gleissberg cycle) and other I stars $(i, p)$ lying near the A branch then suggests that some of the long-term "trends" may actually be segments of cycles with $P_{\text {cyc }} \gg 25 \mathrm{yr}$ on the A branch that are not yet resolved by the Mount Wilson time series. We discuss this further in $\S 4.3$.

Long-term trends of more active stars cannot be explained by this means. They may be segments of longterm aperiodic fluctuations; active stars often do not show clear cycles (Wilson 1978; Bea95). However, we note that an imaginary extension of the $\mathrm{S}$ branch (Figs. 1 and 5, dotted) lies below all limits on $\omega_{\text {cyc }} / \Omega$ (assuming $P_{\text {cyc }} \geq 25 \mathrm{yr}$ ) for active star trends with only one exception (star $\beta$ ). Thus, it seems possible that some trends in active stars may be segments of cycles lying on this S-branch extension, though lacking a concrete example (like the solar Gleissberg cycle) this idea is speculative.

\subsection{Theoretical Implications}

In Paper I we interpreted the ratio $\omega_{\text {cyc }} / \Omega$ as a measure of the $\alpha$-effect. This interpretation was based on a simple model (Robinson \& Durney 1982; Noyes et al. 1984b), which is basically a one-mode truncation of the $\alpha-\Omega$ dynamo equations (e.g., Krause \& Rädler 1980). The main property of our model is that both generative and destructive effects, characterized by the $\alpha$-effect and a decay rate $\tau^{-1}$, can increase with the magnetic field. With the assump-

TABLE 4

Stellar Properties for Active Luminous Stars

\begin{tabular}{|c|c|c|c|c|c|c|c|c|c|c|}
\hline $\begin{array}{l}\text { Type, }{ }^{\mathrm{a}} \\
\text { Symbol }\end{array}$ & Name & $B-V$ & Spectral Type & $\begin{array}{c}\tau_{\mathrm{c}, \mathbf{T}} \\
\text { (days) }\end{array}$ & $\begin{array}{c}P_{\text {rot }} \\
\text { (days) }\end{array}$ & $\begin{array}{l}P_{\mathrm{cyc}} \\
(\mathrm{yr})\end{array}$ & $\begin{array}{l}P_{\mathrm{cyc}}^{(2)} \\
(\mathrm{yr})\end{array}$ & $w$ & References & Notes $^{b}$ \\
\hline $\mathrm{R}, \mathrm{a} \ldots \ldots$ & HD 12545 & 1.21 & $\mathrm{~K} 0 \mathrm{III}+?$ & 14 & 24.3 & 2.7 & $\ldots$ & 1 & 1 & $\mathrm{p}$ \\
\hline $\mathrm{R}, \mathrm{b} \ldots \ldots$ & UX Ari & 0.91 & K0 IV + G5 V & 74 & 6.44 & $0.433 ?$ & $0.070 ?$ & 0.5 & 2 & rp,gd,t2 \\
\hline$R, c \ldots \ldots$. & VY Ari & 0.96 & $\mathrm{~K} 3 \mathrm{IV}+?$ & 52 & 16.4 & 14 & $\ldots$ & 1 & 1 & $\mathrm{p}$ \\
\hline $\mathrm{P}, \mathrm{d} \ldots \ldots$ & V410 Tau & 1.18 & K3 IV-V & 30 & 1.87 & $6-7$ & $\ldots$ & 1 & 1 & $\mathrm{p}$ \\
\hline $\mathrm{R} \ldots \ldots$. & V711 Tau & 0.92 & K1I V+G5 IV & 80 & 2.84 & $16 ?$ & $5.5 ?$ & 0 & 3 & $\mathrm{p}, \mathrm{r}$ \\
\hline $\mathrm{R}, \mathrm{e} \ldots \ldots$. & $\alpha$ Aur & 0.6 & G6 III + F9 III & 4.0: & 8 & 6.05 & $\ldots$ & 1 & 4 & $\mathrm{a}$ \\
\hline$R, f \ldots \ldots$. & CQ Aur & 1.04 & F5+K1I V & 69: & 10.56 & $4-5$ & $\ldots$ & 0.5 & 5 & $\mathrm{p}$ \\
\hline $\mathrm{R}, \mathrm{g} \ldots \ldots$ & $\sigma \mathrm{Gem}$ & 1.12 & $\mathrm{~K} 1 \mathrm{III}+?$ & 54 & 19.4 & 8.5 & $2.7,14.9$ & 1 & $3,6,7$ & $\mathrm{p}, \mathrm{al}$ \\
\hline $\mathrm{R}, \mathrm{h} \ldots \ldots$ & VV Mon & 1.05 & $\mathrm{~K} 0 \mathrm{IV}+\mathrm{G} 2 \mathrm{IV}$ & 48 & 6.05 & $7-8$ & $\ldots$ & 0.5 & 8 & o \\
\hline $\mathrm{R}, \mathrm{i} \ldots \ldots$ & RU Cnc & 1.02 & K1 IV + F5 IV & 54 & 10.14 & $4-5$ & $\ldots$ & 0.5 & 5 & $\mathrm{p}$ \\
\hline $\mathrm{R}, \mathrm{j} \ldots \ldots$ & EI Eri & 0.67 & $\mathrm{G} 5 \mathrm{IV}+?$ & 19 & 1.945 & $\approx 10$ & $\ldots$ & 1 & 1,7 & $\mathrm{p}, \mathrm{al}$ \\
\hline $\mathrm{R}, \mathrm{k} \ldots \ldots$ & HD 106225 & 1.02 & K0 III-IV + ? & 46 & 10.1 & $4-5$ & $\ldots$ & 1 & 1 & $\mathrm{p}$ \\
\hline $\mathrm{R}, 1 \ldots \ldots$. & RS CVn & 0.96 & G9 IV + F4 IV & 70 & 4.79 & 39 & 19.7 & 1 & 9 & $\mathrm{p}, \mathrm{o}$ \\
\hline $\mathrm{R}, \mathrm{m} . . .$. & SS Boo & 0.96 & $\mathrm{~K} 0 \mathrm{IV}+\mathrm{G} 0 \mathrm{~V}$ & 59 & 7.61 & $7-8$ & $\ldots$ & 0.5 & 8 & 0 \\
\hline $\mathrm{R}, \mathrm{n} \ldots \ldots$ & HR 7275 & 1.09 & K2 III-IV + & 60 & 27.9 & 17.5: & $\ldots$ & 0.5 & 7 & al \\
\hline $\mathrm{R}, \mathrm{o} \ldots \ldots$ & RT Lac & 1.14 & G5:+ G9 IV & 58 & 5.07 & $\approx 30$ & $6 ?$ & 0.5 & 3,10 & $\mathrm{p}, \mathrm{o}, \mathrm{t} 2, \mathrm{al}, \mathrm{gd}$ \\
\hline $\mathrm{R} \ldots \ldots$. & HK Lac & 1.08 & $\mathrm{~K} 0 \mathrm{III}+\mathrm{F} 1 \mathrm{~V}$ & 78 & 24.0 & $\approx 7 ?$ & $\ldots$ & 0 & 11 & $0, r$ \\
\hline $\mathrm{R}, \mathrm{p} \ldots \ldots$ & AR Lac & 0.72 & K0 IV + G2 IV & 43 & 1.98 & 36 & 17 & 1 & 12,13 & $\mathrm{p}, \mathrm{o}, \mathrm{ta}$ \\
\hline $\mathrm{R}, \mathrm{q} \ldots \ldots$ & II Peg & 1.01 & $\mathrm{~K} 2 \mathrm{IV}+?$ & 64 & 6.72 & $\approx 10$ & $\approx 40$ & 1 & $3,7,14,15$ & $\mathrm{p}, \mathrm{al}$ \\
\hline $\mathrm{R}, \mathrm{r} \ldots \ldots$. & $\lambda$ And & 1.01 & G8 IV-III + & 68 & 53.9 & 11.1 & $\ldots$ & 1 & 3,16 & $\mathrm{p}$ \\
\hline
\end{tabular}

${ }^{\text {a }}$ Star type: $\mathrm{R}=\mathrm{RS}$ CVn, $\mathrm{P}=$ pre-MS star.

${ }^{\mathrm{b}}$ NoTES. - p: $P_{\text {cyc }}$ from photometry. o: $P_{\text {cyc }}$ from $O-C$ velocity residuals. a: $P_{\text {cyc }}$ from activity modulation. rp: $P_{\text {cyc }}$ from radio polarization; al: $P_{\text {cyc }}$ from active longitude migration. ta: $\left\langle\tau_{c}\right\rangle$ of two stars. t2: $\tau_{c}$ based on combined stars' $B-V$. r: rejected (for HK Lac, see Olah et al. 1997; for V711 Tau, the long $P_{\text {cyc }}$ was $\approx$ time series length, and the short $P_{\text {cyc }}$ was not persuasive on reanalysis). gd: short "cycle" may reflect active region growth/decay.

REFERENCES.- (1) Strassmeier et al. 1997; (2) Massi et al. 1998; (3) Henry et al. 1995; (4) Katsova \& Shcherbakov 1998; (5) Busso et al. 1984; (6) Strassmeier et al. 1988; (7) Berdyugina \& Tuominen 1998; (8) Busso et al. 1985; (9) Rodonò et al. 1995; (10) İbanoğlu et al. 1998; (11) Olah et al. 1986; (12) Jetsu et al. 1997; (13) Lanza et al. 1998a; (14) Bohusz \& Udalski 1981; (15) Hartmann et al. 1979; (16) Hall et al. 1991. 
tions $\alpha \sim \alpha_{0}|B|^{n}$ and $\tau^{-1} \sim \tau_{0}^{-1}|B|^{m}$, the model predicts relations between $m, n$, and the observed exponents $\delta$ (in $\omega_{\text {cyc }} / \Omega \propto \mathrm{Ro}^{-\delta}$; called $\sigma$ in Paper I) and $\mu$ (in $\left\langle R_{\mathrm{HK}}^{\prime}\right\rangle \propto$ $\left.\mathrm{Ro}^{-\mu}\right)$,

$$
\begin{aligned}
& m=(\delta+1) \kappa / \mu, \\
& n=(2 \delta-q) \kappa / \mu,
\end{aligned}
$$

where $\mu \approx 1, \kappa \approx 1 / 2$, and $-2 \leq q \leq 0$ (see Paper I for details). Here, $\kappa$ is defined by $\left\langle R_{\mathrm{HK}}^{\prime}\right\rangle \propto\langle|\boldsymbol{B}|\rangle^{\kappa}$, where $\langle|\boldsymbol{B}|\rangle$ is the average (unsigned) photospheric field strength. The connection with the mean toroidal field (denoted by $B$ ), is unclear; as in Paper I, we assume $|B| \propto\langle|\boldsymbol{B}|\rangle$. We note that the model fails for $m<n / 2$, where the nonlinearity in $\tau$ cannot control the growing $\alpha$-effect for large $|B|$.

We continue to explore the model of Paper I here but allow for new behavior once $|B|$ is very large. We use three discontinuous power laws, so $\alpha$ is written in the form

$$
\alpha(B)= \begin{cases}\alpha_{I}|B|^{n_{I}} & \text { on the I branch } \\ \alpha_{A}|B|^{n_{A}} & \text { on the A branch } \\ \alpha_{S}|B|^{n_{S}} & \text { on the S branch },\end{cases}
$$

where $\alpha_{I}, \alpha_{A}$, and $\alpha_{S}$ are the values of $\alpha_{0}$ on these branches for $|B|=B_{\text {eq }}$, i.e., at some reasonably defined equipartition field strength. We adopt a similar relation for the dissipation rate $\tau^{-1}$, defining the three exponents $m_{I}, m_{A}$, and $m_{S}$. Using equations (1) and (2) and noting $\langle\delta\rangle \approx 0.5$ for the I and A branches, we have $m_{I} \approx m_{A} \approx 3 / 4$ and $1 / 3 \lesssim n_{I} \approx$ $n_{A} \lesssim 3 / 2$. It is not clear that we can use $\mu=1$ on the $S$ branch, since for very active stars $\left\langle R_{\mathrm{HK}}^{\prime}\right\rangle \propto \mathrm{Ro}^{-\mu}$ has saturated and no longer provides a good measure for $\langle|\boldsymbol{B}|\rangle$. More critical in equations (1) and (2) is the ratio $\kappa / \mu$, since $\langle|\boldsymbol{B}|\rangle / B_{\mathrm{eq}} \propto \mathrm{Ro}^{-\mu / \kappa}$. Thus, perhaps $\kappa / \mu \approx 1 / 2$ even for very active stars, since $\langle|\boldsymbol{B}|\rangle$ appears to increase with rotation beyond the point of chromospheric saturation (Saar 1996). If we take $\kappa / \mu \approx 1 / 2$, then $m_{S} \approx 1 / 4$ and $-1 / 2 \lesssim n_{S} \lesssim 1 / 2$. The latter value (for $q=-2$ ) lies on the model's stability boundary $(m=n / 2)$.

For the I and A branches, our new results are consistent with those obtained in Paper I. Despite the uncertainty in $q$, the values of $n_{I}, n_{A}, m_{I}$, and $m_{A}$ are always positive, supporting the idea that both diffusive and generative effects are magnetically driven. This is in clear contrast to the traditional concept of $\alpha-\Omega$ dynamos, where $\alpha$ and $\tau^{-1}$ are caused by thermal convective turbulence and the role of $B$ is solely to quench $\alpha$ and $\tau^{-1}$ once the energy in the field becomes comparable with the turbulent kinetic energy. In fact, the new $\mathrm{S}$ branch is potentially more reminiscent of the conventional quenching concept: while dissipative effects (turbulent diffusion and magnetic buoyancy) still increase with increasing field strengths $\left(m_{S}=1 / 4\right), \alpha$ now decreases with increasing $B$ (for $q>-1$ ).

The uncertainty in the value of $q$ arises from the uncertain $\Omega$-dependence of $\alpha_{0}$ and the radial differential rotation $\Omega^{\prime}$. If we assume $\alpha_{0} \propto \Omega^{q_{\alpha}}$ and $\Omega^{\prime} \propto \Omega^{q \Omega}$, then $q$ is defined as $q=q_{\alpha}+q_{\Omega}-2$. Since $\alpha_{0}$ would be vanishing in the absence of rotation, one might expect $\alpha_{0} \propto \Omega$. However, this must be subject to some quenching for rapid rotation (Kitchatinov, Rüdiger, \& Küker 1994). Likewise, $\Omega^{\prime}$ may increase with $\Omega$ but become constant for rapid rotation. Here, however, we have some observational evidence: study of variations in $P_{\text {rot }}$ over time suggest $0.2 \lesssim q_{\Omega} \lesssim 0.7$ (see $\S$ 4.2). Therefore it is reasonable to assume that both $q_{\alpha}$ and $q_{\Omega}$ are between 0 and 1 , implying $-2 \leq q \leq 0$. On the $\mathrm{S}$ branch rotation is extremely rapid and so $q \approx-2$ appears likely. This would mean $n_{S}=1 / 2$, so even on the $S$ branch $\alpha$ would be enhanced as $B$ increases.

We stress, however, that the idea of "antiquenching"the magnetic enhancement of $\alpha_{0}$ and $\tau_{0}^{-1}$-depends on the assumption $-2 \leq q \leq 0$. For example, Ossendrijver (1997) assumed that $q_{\alpha}=5$ and $q_{\Omega}=-1$, which yields $q=2$, so that $n_{I} \approx n_{A}=-1 / 2$ and $n_{S}=-3 / 2$. In this case $\alpha_{0}$ is indeed quenched, but we consider this choice of $q$ unlikely on physical grounds. Finally, we note that $\Omega^{\prime}$ could also depend on $B$. Since our model depends only on the product $\alpha \Omega^{\prime}$, antiquenching in $\alpha$ could be mimicked by antiquenching of $\Omega^{\prime}$, yielding $n<0$. Again, however, this is unlikely, because the Lorentz force is normally thought to diminish differential rotation.

A more important concern is that the one-mode model adopted here may be unphysical. As discussed in Paper I, a standard dynamo model in one or two dimensions does not reproduce the scaling laws given in equations (1) and (2). Indeed, recent theoretical results argue against any definitive interpretation of cycle periods within the framework of standard dynamo theory. Using a nonlinear twodimensional model with a solar-like geometry (see Tobias 1997), Tobias (1998) finds that $P_{\text {cyc }} \propto D^{\gamma}$, where $D \sim$ $\mathrm{Ro}^{-(q+2)}$ is the dynamo number and $-0.38 \gtrsim \gamma \gtrsim-0.67$ depending on which of several nonlinearities is dominant. This agrees with earlier results of Moss, Tuominen, \& Brandenburg (1990), who found $-0.5 \gtrsim \gamma \gtrsim-0.6$ for both $\alpha$-quenching and magnetic buoyancy as dominant feedback mechanism. In terms of our variables we have $\gamma=-(\delta+1) /(q+2)$. Using $\delta=1 / 2$ and $q=0$ we have $\gamma=-3 / 4$, which is outside the range of values found by Tobias. For $q=-1$ we have $\gamma=-3 / 2$, which is inconsistent with standard dynamo theory, except when the onemode truncation is adopted.

One way the one-mode model could be reconciled is by noting that $\alpha$ and turbulent diffusion coefficients $(\eta)$ are really integral kernels. In Fourier space the convolution becomes a multiplication, and if both $\alpha$ and $\eta$ decrease with increasing wavenumber in a suitable way, the one-mode model is recovered. While there is some evidence for such behavior (Brandenburg \& Sokoloff 1998), this must be regarded as rather exotic. Another possibility is that the nonlinear feedback works with respect to some slowly varying time-averaged field (rather than the instantaneous field), rendering the model essentially linear.

Even in the framework of the one-mode model, however, there remain some puzzles. Why are there several branches? The branches may correspond to regimes in which the $\alpha$ effect is governed by different magnetic instabilities (e.g., the Parker, Balbus-Hawley, and kink instabilities). Alternatively, the relevant saturation mechanism could in some parameter domain arise from feedback on the large-scale motions (Tobias 1998), a mechanism that is known to lead to multiple bifurcation branches in the far-supercritical regime (Muhli et al. 1995). But then how is a given branch accessed? In Paper I we speculated that stars on the A branch might become unstable and jump to the I branch as they age. The fact that $P_{\mathrm{cyc}}$ and $P_{\mathrm{cyc}}^{(2)}$ often lie on opposite branches, however, suggests that the different branches could correspond to different metastable attractors. Stochastic perturbations from turbulent convection (e.g., Schmitt, Schüssler, \& Ferriz-Mas 1996) could cause the 
solution to jump from one branch to the other, possibly even before one cycle is completed. This might explain the erratic aperiodic HK traces ( $\mathscr{V}$ type) and less well-defined $P_{\text {cyc }}$ in more active stars. Modeling is needed to verify this possibility.

\subsection{Comparison with Other Results}

In an early study (after $16 \mathrm{yr}$ of HK data), Noyes et al. (1984b) found that for 13 inactive stars with welldetermined cycles (essentially the inactive stars in Paper I), $P_{\text {cyc }} \propto \mathrm{Ro}^{1.25}$. We find $P_{\text {cyc }} \propto \tau_{c} \mathrm{Ro}^{1.5}$, a fairly similar result. They were the first to notice that the increase in $P_{\mathrm{cyc}}$ with $P_{\text {rot }}$ disagrees with $\alpha$-quenching and suggested magnetic buoyancy may be important.

Ossendrijver (1997) studied a sample of Mount Wilson HK time series again similar to that analyzed in Paper I- primarily stars with FAP grades of good or excellent from Bea95. He found that for the 13 slowly rotating (inactive) stars, $P_{\mathrm{cyc}} \propto \mathrm{Ro}^{2.0 \pm 0.3}$. Using a simple linear mean-field dynamo model, he could reproduce this relation if $\Omega^{\prime} \propto P_{\text {rot }}^{1.1 \pm 0.2}$, and if the $\alpha$ effect scaled as $\alpha \propto \mathrm{Ro}^{-5.1 \pm 0.6}$. The dependence of $P_{\text {cyc }}$ on Ro is somewhat steeper than that found here but is indeed in good agreement with the result of Paper I $\left(P_{\text {cyc }} \propto \mathrm{Ro}^{1.7}\right)$. However, if the surface differential rotation $\Delta \Omega$ is a good proxy for $\Omega^{\prime}$ (as in the Sun; Thompson et al. 1996), his model's exponent for $\Omega^{\prime}$ has the wrong sign. Observations of $P_{\text {rot }}$ changes over time (also taken from Ca II) indicate at the surface $\Delta P_{\text {rot }} \propto P_{\text {rot }}^{1.3}$, or $\Delta \Omega \propto P_{\text {rot }}^{-0.7}$ (Donahue et al. 1996). Study of $P_{\text {rot }}$ caused by spots (Hall 1991a) suggests $\Delta P_{\text {rot }} \propto P_{\text {rot }}^{1.8}$ or $\Delta \Omega \propto P_{\text {rot }}^{-0.2}$. Our results using the model in $\S 4.1$ (Paper I) suggest $\alpha \propto$ $\omega_{\text {cyc }}^{2} / \Delta \Omega$ or $\alpha \propto \mathrm{Ro}^{-2.3}$, rather less steep than Ossendrijver's result.

Baliunas et al. (1996a) proposed another parameterization of the $P_{\mathrm{cyc}}$-rotation relation: a connection between $\omega_{\text {cyc }} / \Omega$ or $\omega_{\text {cyc }}$ and $\Omega$ itself (cf. Noyes et al. 1984b). We show two forms of this relationship for our stellar sample in Figure 6. Though it loses the nondimensional character of the $\omega_{\text {cyc }} / \Omega-\mathrm{Ro}^{-1}$ relation, the $\omega_{\text {cyc }} / \Omega-\Omega$ formulation has the advantage of placing all stars on a single simple relationship, avoiding need for an additional free parameter, $\tau_{c}$. The $\omega_{\text {cyc }}-\Omega$ relationship is also more compatible with conventional $\alpha-\Omega$ dynamos with magnetic quenching.

In Paper I we found that $\omega_{\text {cyc }} / \Omega \propto \Omega^{-1.20}$ with $\sigma_{\text {fit }}=0.13$ dex, a $30 \%$ worse fit than the combined $\mathrm{Ro}^{-1}$ fit to the I and $\mathrm{A}$ branches. The expanded stellar sample here does not greatly change the slope of the relation $\left(\omega_{\text {cyc }} / \Omega \propto \Omega^{-1.09}\right)$, but the fit worsens, and certain drawbacks of the $\omega_{\text {cyc }} / \Omega-\Omega$ formulation become apparent. If we include only primary $P_{\text {cyc }}$ with $w>0$ from Tables 1 and 2, $\sigma_{\text {fit }}=0.31$ dex, and adding $P_{\mathrm{cyc}}^{(2)}$ yields $\sigma_{\mathrm{fit}}=0.32$ dex. The direct correlation yields a similar result, $\omega_{\text {cyc }} \propto \Omega^{-0.09}$ with $\sigma_{\text {fit }}=0.32 \mathrm{dex}$ (for the sample with $P_{\mathrm{cyc}}^{(2)}$ ), suggesting uncertainties in $\omega_{\mathrm{cyc}}$ dominate. Analogous combined fits to the three branches in Figure 5 (6 degrees of freedom; "comb" fits in Table 3) show much less scatter, yielding $\sigma_{\text {fit }}=0.17 \mathrm{dex}$ (no $P_{\mathrm{cyc}}^{(2)}$ ) and $\sigma_{\text {fit }}=0.14$ dex (including $P_{\text {cyc }}^{(2)}$ ).

Thus, the $\omega_{\text {cyc }} / \Omega-\Omega$ parameterization yields significantly greater scatter (a factor of 2 in the log). Beyond this, it has no mechanism to explain long-term trends or $P_{\mathrm{cyc}}^{(2)}$ in stars. It also does not account for the "anomalous" short $P_{\text {cyc }}$ seen in HD 120136 (star Y) and UX Ari (Massi et al. 1998; star b), which lie far above the main relation. While it is not yet clear that these are dynamo cycles - we include neither in the fits - both are potentially explained with our model, since star $\mathrm{b}$ lies on an extension of the I branch and star $\mathrm{Y}$ lies near it (Figs. 1 and 5). The single $\omega_{\text {cyc }}-\Omega$ fit is also poor but has some interesting small-scale structure; we will explore this in a future paper.

\subsection{A Sketch of Dynamo Cycle Period Evolution}

Based on the above results, we can now revise and expand the dynamo evolution scenario proposed in Paper I. Our stellar sample now extends to much larger $\mathrm{Ro}^{-1}$

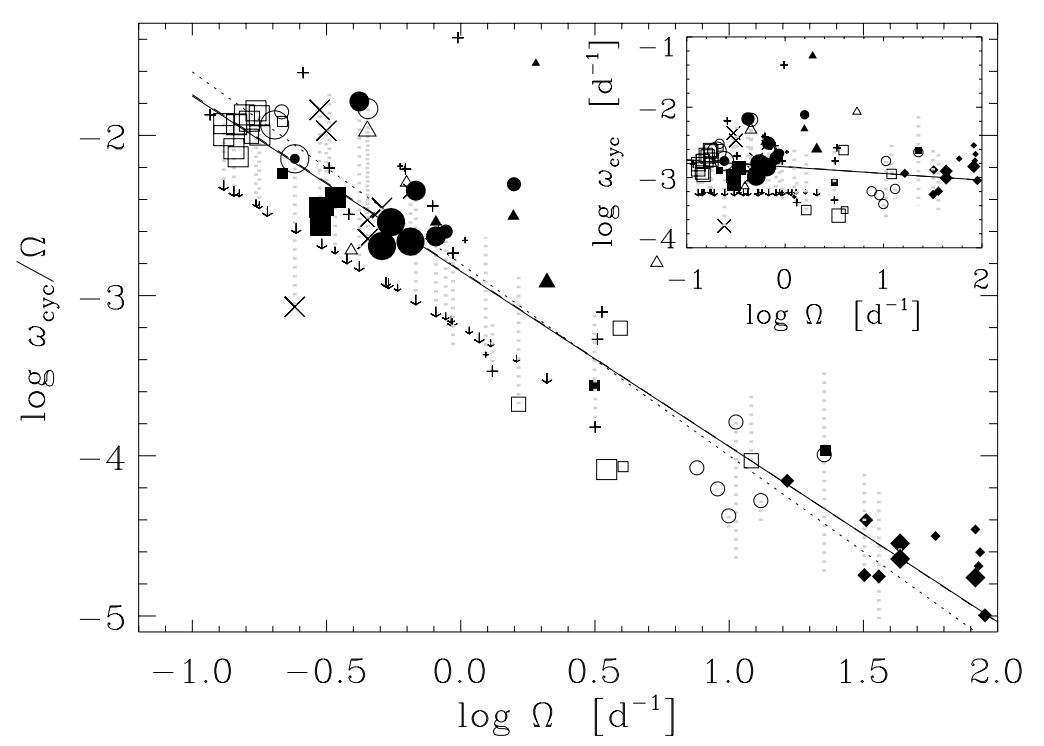

Fig. 6.-Plot showing the correlation between $\omega_{\text {cyc }} / \Omega$ and $\Omega$ for all the stars; symbols as in Fig. 5. If a single fit is made (like Bea96) to all $P_{\text {cyc }}$ with $w>0$ from Tables 1 and 2 and all $P_{\text {cyc }}^{(2)}$ with $w>0$ from Table 1 , we find $\omega_{\text {cyc }} / \Omega \propto \Omega^{-1.09}$ (solid) with $\sigma_{\text {fit }}=0.32$ dex; if $P_{\text {cyc }}^{(2)}$ are excluded, the fit is similar and $\sigma_{\text {fit }}=0.31$ dex. Either fit shows noticeably more scatter than fits with $\mathrm{Ro}^{-1}$ (Table 4 Fig. 5) and fails to explain $P_{\mathrm{cyc}}^{(2)}$ or stars with long-term trends. The dashed line shows the fit for stars in Paper I (and primary $\left.P_{\text {cyc }}\right)$ only $\left(\omega_{\text {cyc }} / \Omega \propto \Omega^{-1.20} ; \sigma_{\text {fit }}=0.13\right.$ dex). The inset shows $\omega_{\text {cyc }}$ vs. $\Omega$ for all stars, which yields an analogous result $\left(\omega_{\text {cyc }} \propto \Omega^{-0.09}, \sigma_{\text {fit }}=0.32 \mathrm{dex}\right.$; solid $)$. 
values, roughly equivalent to much younger ages. For the Wilson survey stars, Donahue (1993) derived a relationship between $t$ and $\left\langle R_{\mathrm{HK}}^{\prime}\right\rangle$ accurate for dwarfs with $\log t \gtrsim 8.5$ (about the age of the UMa stream) given by

$$
\log t=10.725-1.334 R_{5}+0.4085 R_{5}^{2}-0.0522 R_{5}^{3},
$$

where $R_{5}=10^{5} \times R_{\mathrm{HK}}^{\prime}$. This can be easily converted into a $t-\mathrm{Ro}^{-1}$ relation by using an exponential fit to the $\left\langle R_{\mathrm{HK}}^{\prime}\right\rangle-$ $\mathrm{Ro}^{-1}$ relation (see Saar 1998a):

$$
\log t=10.725-7.617 z+13.319 z^{2}-9.718 z^{3},
$$

where $z=\exp (-10.017 \mathrm{Ro})$. (The constants differ from those of Saar (1998a) because of the use of $\tau_{\mathrm{c}, \mathrm{T}}$ in Ro here.) We use these relations to estimate ages for $\log t \gtrsim 8.0$.

For stars much younger than $t \sim 0.3 \mathrm{Gyr}$, however, rotation at fixed $B-V$ is not single-valued, as not all stars have had time to spin down any excess initial angular momentum (e.g., Allain 1998). Thus, $t$ at a given $\mathrm{Ro}^{-1}$ will depend on $B-V$ and initial conditions. Study of the $P_{\text {rot }}$ compiled by O'Dell et al. (1995) and Krishnamurthi et al. (1998) indicates that single stars in the Pleiades $(t \sim 0.07 \mathrm{Gyr})$ exhibit $1.6 \lesssim \log \mathrm{Ro}^{-1} \lesssim 3.2$, (for $0.55 \lesssim B-V \lesssim 1.4$ ), while in the $\alpha$ Per cluster at $t \sim 0.05 \mathrm{Gyr}, 2.2 \lesssim \log \mathrm{Ro}^{-1} \lesssim 3.3$ for $0.7 \lesssim B-V \lesssim 1.3$, with a strong $T_{\text {eff }}$ dependence in both cases. Since the age of $\alpha$ Per is close to the evolutionary peak in rotation, stars with $\log \mathrm{Ro}^{-1} \gtrsim 3.3$ may have no equivalent "rotational age," as they are artificially spun up past the maximum $\Omega$ of "normal" stars. Only once rotation slows to $\log \mathrm{Ro}^{-1} \approx 3.3$, can we (cautiously) translate our $\mathrm{Ro}^{-1}$ values into "rotational" ages. Note however, that most of the stars in our sample with high $\mathrm{Ro}^{-1}$ (Tables 2 and 3) are actually older stars tidally locked at short $P_{\text {rot }}$ by a close companion. We implicitly assume that these close binaries can stand as proxies for young single stars with otherwise similar properties (i.e., we assume that binarity in itself has no effect on the dynamo). Dynamo evolution in close binaries will clearly be driven at a different rate however (mostly by changes in $\tau_{c}$ ), and thus they will not follow the timeline of the evolution scenario sketched here.

If close binary stars and otherwise identical single stars have similar dynamo behavior, young stars $(t \sim 50 \mathrm{Myr})$ begin on the $\mathrm{S}$ branch with $\omega_{\text {cyc }} / \Omega_{\text {rot }}$ increasing as $\mathrm{Ro}^{0.43}$ (Sw fit). Since $v \sin i \propto t^{-0.5}$ approximately (Skumanich $1972)$, this implies that $\omega_{\mathrm{cyc}} / \Omega_{\mathrm{rot}} \sim t^{0.2}$ or $\omega_{\mathrm{cyc}} \sim t^{-0.3}$. Using a simple mean-field dynamo model (e.g., Paper I; $\S 4.1) \alpha \propto \omega_{\text {cyc }}^{2} / \Omega^{\prime} \propto t^{-0.6+q_{\Omega} / 2}$, which for reasonable values of $q_{\Omega}$ (see $\S \S 4.1$ and 4.2 ) yields $\alpha \propto t^{-0.6}$ to $t^{-0.1}$. This corresponds to an $\alpha$ effect slowly decreasing with time as rotation and $\langle B\rangle$ decrease - inconsistent with the traditional idea of $\alpha$-quenching (e.g., $\alpha \propto B^{-2}$; see $\S$ 4.1). Instead, $\alpha$ is enhanced by strong $B$ (see Brandenburg 1998). Surface differential rotation appears to be minimal in rapid rotators (Hall 1991a; Donahue et al. 1996; Donati \& CollierCameron 1997). If this reflects trends in $\Omega^{\prime}$ as well, smallscale processes (e.g., magnetic buoyancy, helicity) are likely more important than large scale shear for dynamo action in these stars.

We have not explored the significance of $P_{\mathrm{cyc}}^{(2)}$ in S-branch stars or included them in fits. They are less certain than the $P_{\text {cyc }}^{(2)}$ from the Mount Wilson data, and some may be spot migration periods or growth/decay timescales (Henry et al. 1995). If some are true dynamo cycles, it is notable they apparently have no preferred locus in the $\omega_{\text {cyc }} / \Omega-\mathrm{Ro}^{-1}$ plane (though statistics are small). Dynamos on the $\mathrm{S}$ branch may thus be more "erratic" in some sense, fluttering chaotically between various quasi-stable $P_{\text {cyc }}$ or holding several $P_{\text {cyc }}$ simultaneously.

Some long-term trends seen in A-branch stars (e.g., HD $1835=\mathrm{A})$ may be segments of secondary $P_{\mathrm{cyc}}$ on the $\mathrm{S}$ branch (or an extension thereof). Though speculative (see $\S 4$ ), if this idea is correct, stars might often show cycles on both $\mathrm{A}$ and $\mathrm{S}$ branches, analogous to the situation between the I and A branches.

In the range $2.0 \leq \log \mathrm{Ro}^{-1} \leq 2.4(t \sim 0.06-0.08 \mathrm{Gyr})$, a sharp transition occurs (with $\omega_{\text {cyc }} / \Omega \propto \mathrm{Ro}^{\delta_{\mathrm{T}}}, \delta_{\mathrm{T}} \sim 4$ ), which takes stars up to the A branch and possibly beyond. Stars $G$ and $H$ document the transition, supported tentatively by many RS CVn's (plus signs). The cause of the transition is not yet clear, but the steep negative exponent $\delta_{\mathrm{T}}$ implies strong $\alpha$-quenching. We note that LQ Hya $(G)$ is single, and thus this rapid change cannot be caused by binary tidal interactions alone.

Once on the A branch, we reach the age-activity realm of Paper I. The larger stellar sample leads to some new insights, however. The presence of a few RS CVns (plus signs) on a possible extension of the I branch suggests that stars can develop $P_{\text {cyc }}$ on the I branch at almost the same time as they arrive on the $\mathrm{A}$ branch, at rotation rates as fast as $\log \mathrm{Ro}^{-1} \sim 1.9(t \sim 0.09 \mathrm{Gyr})$. The first concrete evidence for dwarfs on these branches, however, comes at log $\mathrm{Ro}^{-1} \approx 1.65$ (star $\mathrm{X}, t \sim 0.15 \mathrm{Gyr}$ ) on the A branch, and at $\log \mathrm{Ro}^{-1} \approx 1.1(t \sim 1.3 \mathrm{Gyr})$ on the I branch. The revised correlations between $\omega_{\text {cyc }} / \Omega_{\text {rot }}$ and $\left\langle R_{\mathrm{HK}}^{\prime}\right\rangle$ or $\mathrm{Ro}^{-1}$ are typically less steep and significantly more self-consistent than those in Paper I ( $\$ 3.1$; Table 3). The $2 w$ fits average to $\omega_{\text {cyc }} / \Omega_{\text {rot }} \propto R^{-0.48}$ and $\omega_{\text {cyc }} / \Omega_{\text {rot }} \propto\left\langle R_{\mathrm{HK}}^{\prime}\right\rangle^{0.55}$. This implies that approximately, $\omega_{\text {cyc }} / \Omega_{\text {rot }} \propto t^{-0.25}$ on both branches, or $\omega_{\text {cyc }} \propto t^{-0.75}$. Using Paper I's simple dynamo model once more, $\alpha \propto \omega_{\mathrm{cyc}}^{2} / \Omega^{\prime}$ or $\alpha \propto t^{-1.5}$ to $t^{-1}$. We thus confirm the result of Paper I that $\alpha$ decreases with decreasing rotation as the stars age on the I and A branches, implying that $\alpha$ increases with $B$. The effect is even stronger than on the $\mathrm{S}$ branch, perhaps because of reduced quenching by lower $\langle B\rangle$. Dynamic effects of the buoyant flux tubes increase the helicity (Brandenburg 1998), and we have something akin to the flux tube dynamo of Schüssler (1980) and Schmitt, Schüssler, \& Ferriz-Mas (1996).

Our inclusion of $P_{\mathrm{cyc}}^{(2)}$ and long-term trends of the Sun and stars in this paper puts a new twist on later evolution of the cycle period. Instead of evolving from the A to the I branch, it now appears that stars can have $P_{\text {cyc }}$ on one branch, or the other, or both. In dwarfs, cycles may at first reside only on the A branch, but by $\log \mathrm{Ro}^{-1} \approx 1.1(t \sim 1.5$ Gyr), some stars clearly show cycles on the I branch, and some have cycles on both. Paper I suggested a possible mass dependence for the time of appearance of cycles on the I branch. In the enlarged sample studied here this tendency seems less apparent. However, the branch on which a star has its primary $P_{\text {cyc }}$ does appear to be mass and age dependent. For $\log \mathrm{Ro}^{-1} \leq 1.1$, high-mass $\mathrm{F}$ stars have their primary cycles predominantly on the A branch, while lower mass $\mathrm{K}$ stars dominate primary $P_{\mathrm{cyc}}$ on the I branch. Younger G and K stars tend to have primary $P_{\mathrm{cyc}}$ on the A branch, while older ones have their primary $P_{\text {cyc }}$ on the I branch. Also, as already noted (§3.1), the stars are not strictly segregated by activity on the two branches, particularly when considering secondary periods, which are typi- 
cally on the opposite branch. Thus, the strict segregation of stars by activity between the two branches, and the evolution of stars from A branch to I branch (Paper I) appear to be oversimplifications.

\subsection{Predictions of the Model}

If the general scenario outlined above is correct, we can use the fits to predict $P_{\text {cyc }}$ for stars without clear cycle periods. If the long-term "trends" of inactive stars are actually segments of long-period cycles, we can predict $P_{\text {cyc }}^{(2)}$ assuming the $P_{\text {cyc }}$ will lie on the A branch. Table 1 lists the predicted periods for these stars, denoted $P_{\mathrm{cyc}}^{(\mathscr{L})}$, which were computed using the average of $P_{\mathrm{cyc}}^{(\mathscr{L})}$ determined from the " $2 w$ " fits to $\mathrm{Ro}^{-1}$ (Fig. 1) and $\left\langle R_{\mathrm{HK}}^{\prime}\right\rangle$ (Fig. 2). Excluding one evolved star $(\operatorname{star} x)$, the two estimates agree well, with $\left\langle P_{\mathrm{cyc}}^{(\mathscr{L})}(\mathrm{Ro})-P_{\mathrm{cyc}}^{(\mathscr{L})}(\mathrm{HK})\right\rangle=-2 \pm 6$ yr. If we apply our model to I-branch stars with known $P_{\mathrm{cyc}}^{(2)}$ (stars $a, i$, and $p$ ), we find $\sigma=0.26$ dex (notably, the Sun is poorly fitted). Thus, the $P_{\text {cyc }}^{(\mathscr{L})}$ estimates may be uncertain by $\sim \pm 0.2$ dex, though statistics are small. We have chosen to not compute $P_{\text {cyc }}^{(\mathscr{L})}$ for active stars with trends (by assuming they lie on an extension of the $\mathrm{S}$ branch; $\S 4.3$ ) because of the uncertainty about the reality of such $P_{\mathrm{cyc}}^{(\mathscr{L})}$, and whether the $\mathrm{S}$ branch extends to $\log \mathrm{Ro}^{-1} \lesssim 2.0$ (see $\S 3.2$ ).

We can also use our model to predict $P_{\text {cyc }}$ for "flat activity" stars, assuming they are currently in Maunder-like magnetic minima (Baliunas \& Jastrow 1990; Saar 1998a). Here the estimates are more uncertain, since we can use only the $\omega_{\text {cyc }} / \Omega$ versus Ro ${ }^{-1}$ relation to estimate $P_{\text {cyc }}$. (Use of $\left\langle R_{\mathrm{HK}}^{\prime}\right\rangle$ is inappropriate if these stars' $\mathrm{HK}$ fluxes are reduced by the temporary cessation of cycles.) We have estimated $P_{\text {cyc }}$ for all stars in Bea95 with measured $P_{\text {rot }}$ that are classified $\mathscr{F}$ or have $\Delta S /\langle S\rangle \leq 1.5 \%$ in Table 1 of
Bea95 (Table 5). The $M_{V}$ values implied by Hipparcos $\pi$ indicate that many of these stars are evolved. We therefore assigned revised spectral types (following $\S 2.1$ ) and computed $\mathrm{Ro}^{-1}$ using an evolution-modified $\tau_{\mathrm{c}, \mathrm{T}}$ (GMD; using $\tau_{\mathrm{c}, \mathrm{E}}$ yields similar results). We then estimated $P_{\mathrm{cyc}}$ from the value of $\omega_{\text {cyc }} / \Omega$ on either the I or A branch, using the I $2 w$ and A2w fits. We primarily list only those $P_{\text {cyc }} \geq 4 \mathrm{yr}$, since there is little evidence for shorter periods in the data. The resulting estimates should be within $\sigma_{\text {fit }}(\sim 0.1-0.2$ dex $)$ of $P_{\text {cyc }}$ for those $\mathscr{F}$ stars that emerge from Maunder minimum-like states.

We can also venture to predict $P_{\text {cyc }}$ for young stars in open clusters. While these may not show cycles in $\langle S\rangle$ (see $\mathscr{V}$ HK stars) caused by saturation, photometric (spot) cycles may be detectable, if present (e.g., Table 2 ; § 2.2). We have added a small sample of cluster stars to Table 5 and made $P_{\text {cyc }}$ estimates based on the I $2 w, \mathrm{~A} 2 w$, and $\mathrm{S} 6 w$ fits, assuming cycles exist. Three of these stars (HD 129333, Hz 686, and $\mathrm{Hz} 3163$ ) have recent Doppler images (Strassmeier \& Rice 1998; Stout-Batalha \& Vogt 1996). In some cases, $\mathrm{Ro}^{-1}$ could potentially be on the S-A transitional branch or the (speculative) $\mathrm{S}$ branch extension; the corresponding $P_{\mathrm{cyc}}$ are also given. While none of these stars yet has a clear $P_{\text {cyc }}$ (the 12 yr cycle proposed for HD 129333 by Dorren \& Guinan 1994 seems ruled out by Rea98), we note weak "ripples" in the Ca II $\left(P_{\text {cyc }} \sim 3\right.$ yr $)$ and photometry $\left(P_{\text {cyc }} \sim\right.$ 2 yr) of HD 129333 (see Rea98) that are possibly consistent with a short $P_{\text {cyc }}$ on the A branch.

\section{SUMMARY}

We have extended our study of the evolution of the dynamo cycle period (Paper I) by adding selected photometric cycles from active BY Dra and W UMa systems

TABLE 5

Predicted Cycle Periods for Flat Activity and Cluster Stars

\begin{tabular}{|c|c|c|c|c|c|c|c|c|c|}
\hline Type & HD/Name & $B-V$ & Spectral Type & $M_{V}$ & $\begin{array}{c}\tau_{\mathrm{c}, \mathrm{E}} \\
\text { (days) }\end{array}$ & $\begin{array}{c}\tau_{\mathrm{c}, \mathbf{T}} \\
\text { (days) }\end{array}$ & $\begin{array}{c}P_{\text {rot }} \\
\text { (days) }\end{array}$ & $\begin{array}{c}P_{\text {cyc }} \\
\text { (days) }\end{array}$ & Branch $^{\mathrm{b}}$ \\
\hline$F \ldots \ldots$ & 9562 & 0.64 & $\mathrm{G} 2 \mathrm{IV}^{\mathrm{c}}$ & 3.39 & 11.4 & $14:^{\mathrm{d}}$ & 29 & 49,8 & A,I \\
\hline$F \ldots \ldots$ & 10700 & 0.72 & G8 Vp & 5.68 & 15.1 & 15.8 & 34 & 59,9 & $\mathrm{~A}, \mathrm{I}$ \\
\hline$F \ldots \ldots$ & 45067 & 0.56 & F8 IV-V & 3.28 & 7.7 & $6.7:^{\mathrm{d}}$ & 8 & 10 & A \\
\hline$F \ldots \ldots$ & 89744 & 0.54 & F7 IV ${ }^{c}$ & 2.78 & 6.8 & $3.9:^{\mathrm{d}}$ & 9 & 16 & A \\
\hline$F \ldots \ldots$ & 107213 & 0.50 & F8 IV ${ }^{c}$ & 2.90 & 5.2 & $4.3:^{\mathrm{d}}$ & 9 & 15 & $\mathrm{~A}$ \\
\hline$F \ldots \ldots$ & 124570 & 0.54 & F6 IV & 2.92 & 6.8 & $6.5:^{\mathrm{d}}$ & 26 & 67,9 & A,I \\
\hline$F \ldots \ldots$ & 143761 & 0.60 & G0 Va & 4.18 & 9.5 & 10.2 & 17 & 25,4 & A,I \\
\hline$F \ldots \ldots$ & 178428 & 0.70 & G4 IV-V ${ }^{c}$ & 4.47 & 14.2 & $15:^{\mathrm{d}}$ & 22 & 30,5 & A,I \\
\hline$F \ldots \ldots$ & 207978 & 0.42 & F0 V & 3.31 & 2.6 & 1.5 & 3 & 5 & A \\
\hline$F \ldots \ldots$ & 212754 & 0.52 & F5 IV ${ }^{c}$ & 2.78 & 6.0 & $3.0^{\mathrm{d}}$ & 12 & 30,4 & A,I \\
\hline $\mathrm{H} . . . .$. & 28099 & 0.68 & G6 V & $\ldots$ & 13.3 & 14.2 & 8.7 & $7,79 ?$ & $\mathrm{~A}, \mathrm{~S}$ \\
\hline $\mathrm{H} \ldots \ldots . .$. & 28783 & 0.88 & K1 V & $\cdots$ & 21.3 & 20.3 & 9.36 & $6,96 ?$ & A,S \\
\hline $\mathrm{H} . . . .$. & 285805 & 1.17 & $\mathrm{~K} 5 \mathrm{~V}$ & $\ldots$ & 25.0 & 28.9 & 11.92 & $8,130 ?$ & $\mathrm{~A}, \mathrm{~S}$ \\
\hline U...... & 72905 & 0.62 & G1.5 V & 4.86 & 10.4 & 11.6 & 4.68 & $3,51 ?$ & $\mathrm{~A}, \mathrm{~S}$ \\
\hline$P \ldots \ldots$ & 129333 & 0.63 & G1 V & 4.95 & 10.9 & 12.1 & 2.8 & $1.4 ?, 39 ?$ & A,S \\
\hline$P \ldots \ldots$ & Hz 314 & 0.62 & G1 V & $\ldots$ & 10.4 & 11.6 & 1.05 & $5:, 22$ & $\mathrm{~T}, \mathrm{~S}$ \\
\hline$P \ldots \ldots$ & Hz 3163 & 1.01 & $\mathrm{~K} 5 \mathrm{~V}$ & $\ldots$ & 24.3 & 23.9 & 0.42 & 18 & $S$ \\
\hline$P \ldots \ldots$ & Hz 686 & 1.39 & M0 V & $\ldots$ & 25.0 & 42.9 & 0.40 & 22 & S \\
\hline A ......... & HE 699 & 0.71 & $\sim \mathrm{G} 5 \mathrm{~V}$ & $\cdots$ & 14.7 & 15.3 & 0.33 & 13 & S \\
\hline A ...... & HE 86 & 1.32 & $\sim \mathrm{K} 7 \mathrm{~V}$ & $\ldots$ & 25.0 & 37.5 & 0.21 & 15 & $S$ \\
\hline
\end{tabular}

a Star type: F: flat activity (in Maunder-like minimum?). H: Hyades $(t \approx 0.5 \mathrm{Gyr})$. U: UMa stream $(t \approx 0.3 \mathrm{Gyr})$. P: Pleiades $(t \approx 0.07 \mathrm{Gyr})$. A: $\alpha$ Per $(t \approx 0.05 \mathrm{Gyr})$.

b I, A, S, T: on inactive, active, superactive or approximate transition branches (using $\tau_{\mathrm{c}, \mathrm{T}}$ ).

${ }^{c}$ Luminosity class adjusted based on $M_{V}$.

${ }^{\mathrm{d}} \tau_{c}$ value adjusted (GMD) for evolution. 
(§ 2.2), cycles of various types found in $\mathrm{CV}$ secondaries $(\S$ 2.3) and lower FAP graded cycles and long-term trends from the Mount Wilson HK monitoring program (Bea95; Rea98). In the process we have developed a new empirical $\tau_{c}$ and a new $\left\langle R_{\mathrm{HK}}^{\prime}\right\rangle-\mathrm{Ro}^{-1}$-age relation and have estimated new $M_{V}$ and luminosity classes for much of our sample.

Adding these stars confirms some of the basic results of Paper I, namely, that for $\log \mathrm{Ro}^{-1} \lesssim 1.8$ (ages $t \gtrsim 0.1 \mathrm{Gyr}$ ), stars lie on two nearly parallel branches, separated by a factor of $\sim 6$ in $P_{\text {cyc }}$, with $\omega_{\text {cyc }} / \Omega \propto \mathrm{Ro}^{-0.5}$ and $\omega_{\text {cyc }} / \Omega \propto$ $\left\langle R_{\mathrm{HK}}^{\prime}\right\rangle^{0.5}$. More active stars typically populate the lower $\omega_{\text {cyc }} / \Omega(\mathrm{A})$ branch, and as stars age, lower mass stars tend to have their primary $P_{\text {cyc }}$ in the upper $\omega_{\text {cyc }} / \Omega$ (I) branch. Many secondary cycle periods lie on the opposite branch from the primary $P_{\text {cyc }}$. The new branch fits are more internally consistent than in Paper I.

If close binaries (BY Dra and W UMa variables, CV secondaries) can be used as proxies for young, rapidly rotating single stars, the (mostly photometric) cycles of these stars populate a new "superactive" (S) branch with the opposite slope $\left(\omega_{\text {cyc }} / \Omega \propto \mathrm{Ro}^{0.4}\right)$ lying below the I and A branches. A few active dwarfs and many RS CVn variables appear to delineate a transitional zone between the $S$ branch and the others; a few RS CVns and single subgiants populate the I and A branches. Thus evolved stars also appear generally consistent with the results for dwarfs (though the close binaries will evolve differently).

We interpret the results using an extension of the simple model used in Paper I. Within this framework, the changes in $\omega_{\text {cyc }} / \Omega$ we observe may be driven by changes in $\alpha$ effect with rotation rate. From a high- $B$ regime (S branch) in young/rapid rotators $(t \lesssim 0.1 \mathrm{Gyr})$, where $\alpha$ is a weakly increasing function of $B$ (S branch), the dynamo undergoes a strongly $\alpha$-quenched transition to a regime in which the enhancement of $\alpha$ by magnetic feedback effects is even stronger. Here the dynamo is also partly unstable, showing two favored solutions (I and A branches) and sometimes both simultaneously $\left(P_{\mathrm{cyc}}^{(2)}\right)$. Results of another recent model (Tobias 1998) support the idea of $\omega_{\text {cyc }}$ evolution because of changes in the dominant nonlinear quenching effects but argue that a definitive interpretation in terms of evolution in $\alpha$ alone may be an oversimplification.

Models correlating $\omega_{\text {cyc }} / \Omega$ or $\omega_{\text {cyc }}$ with $\Omega$ (e.g., Baliunas et al. 1996a), while more compatible with conventional $\alpha-\Omega$ dynamos and placing all stars on a single relation, show significantly more scatter (a factor of 2 in the $\log$ ). Such models also have no clear explanation for $P_{\text {cyc }}^{(2)}$, longterm trends or very short $P_{\text {cyc }}$ stars (e.g., UX Ari).

We use our models to predict $P_{\text {cyc }}$ for long-term trends seen in inactive stars $\left(=P_{\text {cyc }}^{(\mathscr{L})} ;\right.$ Table 1$)$ assuming the trends are segments of long $(>25 \mathrm{yr}$ ) cycles. We also list in Table 5 the predicted $P_{\text {cyc }}$ for "flat activity" stars (assuming they are in Maunder-like minima) and for a small sample of young cluster stars (assuming they have cyclic dynamos).

We are grateful to the referee for helpful suggestions and to Sallie Baliunas, Ed DeLuca, Bob Donahue, Jeff McClintock, Fred (Ni!) Ringwald, and Steve Tobias for enlightening discussions. S. H. S. was supported by NSF grant AST-9528563 and thanks the University of Newcastle, where some of this work was completed, for their support.
Allain, S. 1998, A\&A, 333, 629

Alonso, A., Arribas, S., \& Martinez-Roger, C. 1996, A\&A, 313, 873

Applegate, J. H. 1992, ApJ, 385, 621

Bakos, G. A. 1984, AJ, 89, 1740

Baliunas, S. L., et al. 1995, ApJ, 438, 269 (Bea95)

Baliunas, S. L., \& Jastrow, R. 1990, Science, 348, 520

Baliunas, S. L., Nesme-Ribes, E., Sokoloff, D., \& Soon, W. H. 1996a, ApJ, 460, 848

Baliunas, S. L., Sokoloff, D., \& Soon, W. H. 1996b, ApJ, 457, L99

Baliunas, S. L., \& Vaughan, A. H. 1985, ARA\&A, 23, 379

Berdyugina, S., \& Tuominen, I. 1998, A\&A, 336, L25

Bergeat, J., Lunel, M., Sibille, F., \& Van't Veer, F. 1972, A\&A, 17, 215

Bessell, M. S. 1991, AJ, 101, 662

Bianchini, A. 1987, Mem. Soc. Astron. Italiana, 58, 245 1990, AJ, 99, 1941

1992, in ASP Conf. Ser. 29, Vina del Mar Workshop on Cataclys-

mic Variable Stars, ed. N. Vogt (San Francisco: ASP), 284

Bohusz, E., \& Udalski, A. 1981, Acta Astron., 31, 185

Bondar', N. I. 1995, A\&AS, 111, 259

Bradstreet, D. H., Devita, M. J., Hargis, J. R., Jewett, F. D., Lake, J. S., Guinan, E. F., \& Glowina, Z. 1997, BAAS, 29, 1280

Bradstreet, D. H., \& Guinan, E. F. 1990, in Active Close Binaries, ed. C. İbanoğlu (Dordrecht: Kluwer), 467

Brandenburg, A. 1998, in ASP Conf. Ser. 154, Cool Stars, Stellar Systems, and the Sun, ed. R. A. Donahue \& J. A. Bookbinder (San Francisco: ASP), 173

Brandenburg, A., Saar, S. H., \& Turpin, C. J. 1998, ApJ, 498, L51 (Paper I).

Brandenburg, A., \& Sokoloff, D. 1998, Geophys. Astrophys. Fluid Dyn., submitted

Burke, E. W., Fried, R. E., Hall, D. S., Casado, M., Hampton, M., \& Hunt, M. 1992, Inf. Bull. Variable Stars 3722

Busso, M., Scaltriti, F., Blanco, C., Catalano, S., Rodonó, M., Marilli, E., \& Pazzani, V. 1984, A\&A, 135, 255

Busso, M., Scaltriti, F., \& Cellino, A. 1985, A\&A, 148, 29

Dhillon, V. S., Jones, D. H. P., \& Marsh, T. R. 1994, MNRAS, 246, 859

Donahue, R. A. 1993, Ph.D. thesis, New Mexico State Univ. 1996, in IAU Symp. 176, Stellar Surface Structure, ed. K. G. Strassmeier \& J. L. Linsky (Dordrecht: Kluwer), 261

Donahue, R. A., \& Baliunas, S. L. 1992, Sol. Phys., 141, 181

Donahue, R. A., Dobson, A. K., \& Baliunas, S. L. 1997, Sol. Phys., 171, 191

Donahue, R. A., Saar, S. H., \& Baliunas, S. L. 1996, ApJ, 466, 384

Donati, J.-F., \& Cameron, A. C. 1997, MNRAS, 291, 1

\section{REFERENCES}

Dorren, J. D., \& Guinan, E. F. 1994, ApJ, 428, 805

Eaton, J. A., Henry, G. W., \& Fekel, F. K. 1996, ApJ, 462, 888

ESA. 1997, The Hipparcos and Tycho Catalogs (ESA SP-1200) (Noordwijk: ESA)

Flower, P. J. 1996, ApJ, 469, 355

Foukal, P. 1998, ApJ, 500, 958

Frieboes-Conde, H., \& Herczeg, T. 1973, A\&AS, 12, 1

Gleissberg, W. 1971, Sol. Phys., 21, 240

Gray, D. F., \& Baliunas, S. L. 1994, ApJ, 427, 1042 .1995, ApJ, 441, 436

Gunn, A. G., Mitrou, C. K., \& Doyle, J. G. 1998, MNRAS, 296, 150 (GMD)

Hall, D. S. 1990, in Active Close Binaries, ed. C. İbanoğlu (Dordrecht: Kluwer), 95

. 1991a, in The Sun and Cool Stars: Activity, Magnetism, Dynamos, ed. I. Tuominen, D. Moss, \& G. Rüdiger (Berlin: Springer), 353 1991b, ApJ, 380, L85

Hall, D. S., et al. 1991, J. Astrophys. Astron., 12, 281

Hartmann, L., Bopp, B. W., Dussault, M., Noah, P. V., \& Klimke, A. 1981, ApJ, 249, 662

Hartmann, L., Londono, C., \& Phillips, M. J. 1979, ApJ, 229, 183

Heckert, P. A., Maloney, G. V., Stewart, M. C., Ordway, J. I., Hickman, M. A., \& Zeilik, M. 1998, AJ, 115, 1145

Henry, G. W., Eaton, J. A., Hamer, J., \& Hall, D. S. 1995, ApJS, 97, 513

İbanoğlu, C., Keskin, V. Akan, M. C., Evren, S., \& Tunca, Z. 1994, A\&A, 281,811

İbanoğlu, C., Pekünlü, E. R., Keskin, V., Tunca, Z., Evren, S., \& Akan, M. C. 1998, Ap\&SS, 257,11

Jetsu, L., Panago, I., Moss, D., Rodonò, M., Lanza, A. F., \& Tuominen, I. 1997, A\&A, 326, 698

Karimie, M. T. 1983, Ap\&SS, 92, 53

Kaszás, G., Vinkó, K., Szatmáry, K., Hegedüs, T., Gál, J., Kiss, L. L., \& Borkovits, T. 1998, A\&A, 331, 231

Katsova, M. M., \& Shcherbakov, A. G. 1998, A\&A, 329, 1080

King, A. R., Frank, J., Kolb, U., \& Ritter, H. 1996, ApJ, 467, 761

Kitchatinov, L. L., Rüdiger, G., \& Küker, M. 1994, A\&A, 292, 125

Kolb, U., King, A. R., \& Ritter, H. 1998, MNRAS, 298, L29

Krause, F., \& Rädler, K.-H. 1980, Mean-Field Electrodynamics and Dynamo Theory (Oxford: Pergamon Press)

Krishnamurthi, A., et al. 1998, ApJ, 493, 914

Lanza, A. F., Catalano, S., Cutispoto, G., Pagano, I., \& Rodonò, M. 1998a, A\&A, 332, 541

Lanza, A. F., Rodonò, M., \& Rosner, R. 1998b, MNRAS, 296, 893 
Leggett, S. K., Allard, F., Berrimann, G., Dahn, C. C., \& Hauschildt, P. H. 1996, ApJS, 104, 117

Liu, X., Wang, G., Guo, Z., \& Jia, G. 1984, Chin. Astron. Astrophys., 8, 126

Lockwood, G. W., Skiff, B. A., \& Radick, R. R. 1997, ApJ, 485, 789

Maceroni, C., Bianchini, A., Van't Veer, F., Rodonò, M., \& Vio, R. 1989, A\&A, 237, 395

Massi, M., Neidhöfer, J., Torricelli-Ciamponi, G., \& Chuideri-Drago, F. 1998, A\&A, 322, 149

Maulik, D., Donahue, R. A., \&Baliunas, S. L. 1997, Persistent Sub-Yearly Chromospheric Variations in Lower Main-Sequence Stars: $\tau$ Boo and $\alpha$ Com (SAO Tech. Rep).

Mavridis, L. N., Asteriadis, G., \& Mahmoud, F. M. 1982, in Compendium in Astronomy, ed. E. G Mariolopoulos, P. S. Theocaris, \& L. N. Mavridis (Dordrecht: Reidel), 253

Mavridis, L. N., \& Avgoloupis, S. 1986, A\&A, 154, 171

Moss, D., Tuominen, I., \& Brandenburg, A. 1990, A\&A, 228, 284

Muhli, P., Brandenburg, A., Moss, D., \& Tuominen, I. 1995, A\&A, 296, 700

Noyes, R. W., Hartmann, L., Baliunas, S. L., Duncan, D. K., \& Vaughan, A. H. 1984a, ApJ, 279, 763 (Nea84)

Noyes, R. W., Weiss, N. O., \& Vaughan, A. H. 1984b, ApJ, 287, 769

O'Dell, M. A., Panagi, P., Hendry, M. A., \& Collier Cameron, A. 1995, A\&A, 294, 715

Olah, K., Hall, D. S., Boyd, L. J., Genet, R. M., \& Fried, R. E. 1986, Astrophys. Lett., 25, 133

Olah, K., Kövári, Zs., Bartus, J., Strassmeier, K. G., Hall, D. S., \& Henry, G. W. 1997, A\&A, 321, 811

Oprescu, G., Dumitrescu, A., Rovithis, P., \& Rovithis-Livaniou, H. 1996, Inf. Bull. Variable Stars, 4307

Ossendrijver, A. J. H. 1997, A\&A, 323, 151

Patterson, J. 1984, ApJS, 54, 443

Pettersen, B. R., Olah, K., \& Sandmann, W. H. 1992, A\&AS, 96, 497

Phillips, M. J., \& Hartmann, L. 1978, ApJ, 224, 182

Pojmanski, G., \& Geyer, E. H. 1990, Acta Astron., 40, 245

Press, W. H. 1978, Comments Astrophys., 7, 103

Radick, R. R., Lockwood, G. W., \& Baliunas, S. L. 1990, Science 247, 39

Radick, R. R., Lockwood, G. W., Skiff, B. A., \& Baliunas, S. L. 1998, ApJS, 118,239 (Rea98)

Rädler, K.-H. 1986, Astron. Nachr., 307, 89

Reinsch, K. 1994, A\&A, 281, 108

Richman, H. R., Applegate, J. H., \& Patterson, J. 1994, PASP, 106, 1075

Robinson, E. L., Barker, E. S., Cochran, A. L., Cochran, W. D., \& Nather, R. E. 1981, ApJ, 251, 611

Robinson, R. D., \& Durney, B. R. 1982, A\&A, 108, 322

Rodonò, M., \& Cutispoto, G. 1992, A\&AS, 95, 55

Rodonò, M., Lanza, A. F., \& Catalano, S. 1995, A\&A, 301, 75

Rubenstein, E. P., Patterson, J., \& Africano, J. L. 1991, PASP, 103, 1258
Rutten, R. G. M. 1987, Ph.D. thesis, Utrecht

Saar, S. H. 1996, in IAU Colloq. 153, Magnetodynamic Phenomena in the Solar Atmosphere-Prototypes of Stellar Magnetic Activity, ed. Y. Uchida, T. Kosugi, \& H. S. Hudson (Dordrecht: Kluwer), 367 1998a, in ASP Conf. Ser. 154, Cool Stars, Stellar Systems, and the Sun, ed. R. A. Donahue \& J. A. Bookbinder (San Francisco: ASP), 211 1998b, Inf. Bull. Variable Stars 4580, 1

Saar, S. H., \& Baliunas, S. L. 1992, in ASP Conf. Ser. 27, The Solar Cycle, ed. K. Harvey (San Francisco: ASP), 150

Scargle, J. D. 1982, ApJ, 263, 875

Schmitt, D., Schüssler, M., \& Ferriz-Mas, A. 1996, A\&A, 311, L1

Schüssler, M. 1980, Nature, 288, 150

Shengbang, Q., Quingyao, L., \& Yulan, Y. 1998, Ap\&SS, 257, 1

Skumanich, A. 1972, ApJ, 171, 565

Smak, J. 1993, Acta Astron., 43, 121

Smith, D. A., \& Dhillon, V. 1998, MNRAS, 301, 767

Soon, W. H., Baliunas, S. L., \& Zhang, Q. 1993, ApJ, 414, L33

Stepień, K. 1989, A\&A, 210, 273

Stout-Batalha, N. M., \& Vogt, S. S. 1996, in IAU Symp. 176, Stellar Surface Structure, ed. K. G. Strassmeier \& J. L. Linsky (Dordrecht: Kluwer), 337 Strassmeier, K. G., et al. 1988, A\&A, 192, 135

Strassmeier, K. G., Bartus, J., Cutispoto, G., \& Rodonò, M. 1997, A\&AS, 125,11

Strassmeier, K. G., Hall, D. S., Boyd, L. J., \& Genet, R. M. 1989, ApJS, 69, 141

Strassmeier, K. G., Hall, D. S., Fekel, F. C., \& Scheck, M. 1993, A\&AS, 100, 173

Strassmeier, K. G., \& Rice, J. B. 1998, A\&A, 330, 685

Thompson, M. J., et al. 1996, Science, 272, 1300

Tobias, S. M. 1997, Geophys. Astrophys. Fluid Dyn., 86, 287 1998, MNRAS, 296, 653

Tout, C., \& Pringle, J. E. 1992, MNRAS, 256, 269

Tuominen, I., Rüdiger, G., \& Brandenburg, A. 1988, in Activity in Cool

Star Envelopes, ed. O. Havnes, B. R. Pettersen, J. H. M. M. Schmitt, \& J. E. Solheim (Dordrecht: Kluwer), 13

Vaughan, A. H., \& Preston, G. W. 1980, PASP, 92, 385

Verbunt, F., \& Zwaan, C. 1981, A\&A, 100, L7

Vilhu, O. 1984, A\&A, 133, 117

Vivekananda Rao, P., Sarma, M. B. K., \& Prakash Rao, B. V. N. S. 1991, J. Astrophys. Astr., 12, 225

Warner, B. 1988, Nature, 336, 129

Wilson, O. C. 1978, ApJ, 226, 379

Wolf, S., Mantel, K. H., Horne, K., Barwig, H., Schoembs, R., \& Baernbanter, O. 1993, A\&A, 273, 160

Wolff, C. L. 1976, ApJ, 205, 612

Zangrilli, L., Tout, C. A., \& Bianchini, A. 1997, MNRAS, 289, 59 